\author{
Pierangelo CrucitTi* - Davide Brocchieri* - Francesco BubBiCo* - Paolo CastellucCiO* - \\ Francesco CERVoni* - Edoardo Di RUSSO* - Federica EMILIANI* - Marco Giardini* - Edoardo Pulvirenti*
}

\title{
Checklist di alcuni gruppi selezionati dell'entomofauna del Parco Naturale Archeologico dell'Inviolata (Guidonia Montecelio, Roma)
}

\author{
XLI contributo allo studio della biodiversità della Campagna Romana a nord-est di Roma
}

\begin{abstract}
Riassunto: Sono riportati i risultati di una indagine conoscitiva sistematica effettuata negli anni 2016-2019 su alcuni gruppi di Insecta appartenenti a Odonata, Orthopteroidaea, Dermaptera, Coleoptera, Lepidoptera, Isoptera e Mecoptera monitorati nel Parco Naturale Archeologico dell'Inviolata (Roma, Lazio). Sono descritti i principali caratteri geomorfologici, climatologici e vegetazionali dell'area studiata. I campionamenti sono stati effettuati con metodologie diversificate; raccolta manuale, trappole a caduta, sorgenti luminose, ispezione di feci, animali morti e vegetazione acquatica. È stata accertata la presenza di 533 taxa appartenenti a 101 famiglie. L'ordine maggiormente rappresentato è quello dei Coleoptera (359 taxa) cui appartiene la famiglia più rappresentata, quella dei Carabidae (77 taxa); seguono i Lepidoptera con 107 taxa. Nel complesso, le specie endemiche italiane e/o rare sono numerose. Eriogaster catax ed Euplagia quadripunctaria sono protette dalla Direttiva Habitat (92/43/CEE). Si segnalano in particolare il rinvenimento di Labia minor (Dermaptera), osservata per la prima volta nella Campagna Romana, e di Anthaxia lucens (Buprestidae), per la quale il Parco dell'Inviolata è l'unica stazione nota nel Lazio. L'analisi biogeografica, basata sui corotipi delle specie di Odonata e Coleoptera Carabidae, ha evidenziato la predominanza di elementi ad ampia distribuzione, seguiti da quelli europei e mediterranei. Sono quindi state effettuate comparazioni con l'entomofauna di aree della Campagna Romana a nord-est di Roma. È stata sottolineata l'importanza ecologica dei piccoli bacini lacustri a regime idrologico variabile presenti nell'area protetta.
\end{abstract}

\begin{abstract}
Checklist of selected groups of Insects from the Inviolata Natural Archaeological Park (Guidonia Montecelio, Rome).
The results of a systematic survey carried out during the years 2016-2019 on selected groups of Insects belonging to Odonata, Orthopteroidaea, Dermaptera, Coleoptera, Lepidoptera, Isoptera and Mecoptera, inside the Inviolata Archaeological Natural Park (Rome, Latium), are presented and discussed. The main geomorphological, climatological and vegetational features of the studied area, are described. Sampling was carried out using different methods; manual pick up, traps, light sheets, monitoring of faeces, dead animals and aquatic vegetation. The inventory is represented by 533 taxa belonging to 101 families, all of which characterized from phenological point of view. Coleoptera is the most represented order with 359 taxa, to which Carabidae is the most represented family (77 taxa); the Lepidoptera with 107 taxa follow. Endemic and rare species are numerous. Eriogaster catax and Euplagia quadripunctaria are protected by the Directive 92/43/EEC. Conspicuous discoveries are that of Labia minor (Dermaptera), observed for the first time in the Roman Campagna north-east of the Rome city area, and of Anthaxia lucens (Buprestidae), for which the Inviolata Park is the only known site for Latium. The biogeographical analysis based on the chorotypes is carried out for Odonata and Carabidae; the predominance of species with large distribution, followed by the European and Mediterranean ones is highlighted. Comparisons are made with the entomofauna of other areas of the Roman Campagna. The ecological importance of some pools and ponds with variable hydrological regime inside this protected area has been mentioned.
\end{abstract}

Key words: Insecta, Inviolata Natural and Archaeological Park, Rome, Latium, checklist, conservation.

\section{INTRODUZIONE}

Il Parco Naturale Archeologico dell'Inviolata di Guidonia (provvedimento istitutivo L.R. 22/96, EUAP1032) è un' area protetta ubicata a nord-est dell'insediamento urbano di Roma, limitrofa alle riserve naturali Nomentum (EUAP1039) e Macchia di Gattaceca e Macchia del Barco (EUAP1040) (Locasciulli et al., 1999). Questa riserva è inserita nel cosiddetto "arcipelago mentanese - cornicolano" s.l., settore della Campagna Romana a nord-est di Roma (comuni di
Fonte Nuova, Mentana, Monterotondo, Sant'Angelo Romano, Guidonia Montecelio) la cui denominazione è basata sui seguenti criteri, parzialmente inclusivi: i) piccole aree protette o comunque di rilevante interesse naturalistico immerse in un contesto fortemente antropizzato; ii) frammenti boschivi immersi in una matrice di agrosistemi ed edificato sparso/compatto (Frank \& Lorenzetti, 2005; Lorenzetti \& Battisti, 2006, 2007; Crucitti, 2013).

A partire dal 1997 il sistema delle piccole aree

\footnotetext{
*Pierangelo Crucitti, Davide Brocchieri, Francesco Bubbico, Paolo Castelluccio, Francesco Cervoni, Edoardo Di Russo, Federica Emiliani, Marco Giardini, Edoardo Pulvirenti, Società Romana di Scienze Naturali SRSN, Campus di Villa Esmeralda, Via Fratelli Maristi 43, 00137 Roma, Italia. E-mail: info@srsn.it.
} 
protette della Campagna Romana a nord-est di Roma è stato oggetto di ricerche intensive della Società Romana di Scienze Naturali (SRSN) sulla biodiversità animale con la pubblicazione di checklist ragionate di specie (Crucitti et al., 2013, 2015, 2016). Nel contesto, il Parco Naturale Archeologico dell'Inviolata (PNA Inviolata) è stato oggetto di ricerche sulla biodiversità floristica e faunistica in convenzione con il Parco Regionale dei Monti Lucretili al quale è stato affidato in gestione nel 2016 (Cervoni et al., 2018).

La presente indagine, inserita nella linea di ricerca SRSN "Struttura di zoocenosi di aree protette dell'Italia centrale", si propone di fornire la checklist annotata delle specie di gruppi selezionati di Insecta Odonata, Orthopteroidaea, Dermaptera, Isoptera, Coleoptera, Lepidoptera, Mecoptera - dell'area. Sul PNA Inviolata non risultano ricerche specifiche in merito; dati sull'entomofauna della Città di Roma e aree circostanti, che interessano pertanto marginalmente territori limitrofi al parco, sono discussi in Zapparoli (1997) (Fig. 1).

\section{Materiali e Metodi}

\section{Area di studio}

Il PNA Inviolata è un'area planiziale (50-120 m s.l.m., media $80 \mathrm{~m}$ ) di circa 460 ha (520 ha sino al 2005) compresa tra le frazioni Marco Simone - Laghetto di Guidonia Montecelio e Santa Lucia di Fonte Nuova (Calamita, 2005, 2012; Crucitti et al., 2017). Si tratta di un settore della grande area dell'agro romano nord-orientale tra la Valle dell'Aniene a sud, i

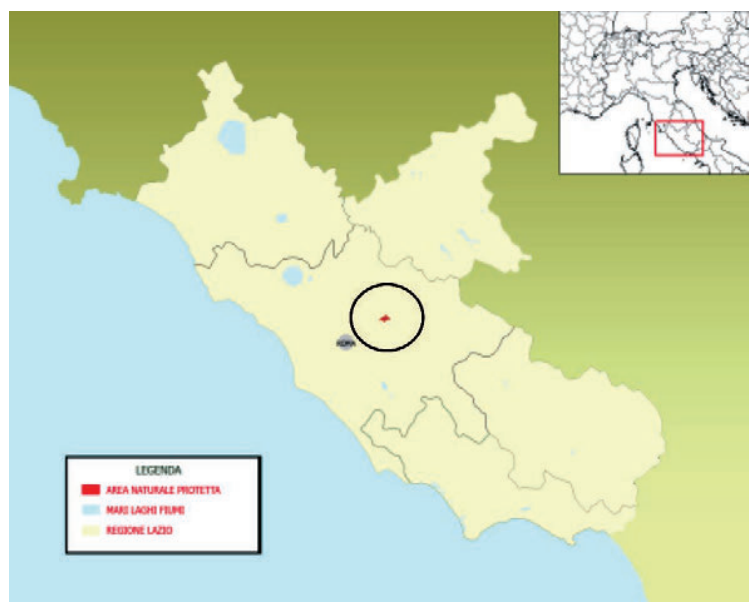

Fig.1. Posizione del PNA Inviolata nel Lazio e nella Provincia di Roma.
Monti Cornicolani a nord, il bacino in travertino delle Acque Albule ad est e l'arco collinare Formello - Tor de Sordi - Castell'Arcione ad ovest (Fig. 1). Il territorio, i cui confini circoscrivono un perimetro irregolare, è delimitato a nord dal Fosso Capaldo, ad est dall' Autostrada A1 tratto Fiano - San Cesareo, ad ovest dall'abitato di Marco Simone Vecchio, a sud dalla Via di Casal Bianco (Fig. 2). L'evoluzione geomorfologica dell'area risulta essenzialmente condizionata dalle fasi erosive dovute ad eustatismo succedutesi nel corso del Quaternario. Il substrato risulta modellato soprattutto in funzione dei materiali costituenti. I dossi sono formati da sedimenti di origine vulcanica, essenzialmente tufo litoide e tufo grigio, meno suscettibili all'erosione delle sabbie e delle argille sabbiose che costituiscono $i$ versanti e le fasce adiacenti. La natura dei terreni affioranti, poco permeabili, e la pendenza, permettono un discreto ruscellamento delle acque meteoriche favorendo il drenaggio verso i fossi principali i quali, in occasione di eventi consistenti, aumentano considerevolmente la portata (Bunone, 2005).

Dalle medie dei dati relative al trentennio 19712000 (Atlante Climatico d'Italia del Servizio Meteorologico dell'Aeronautica Militare; Stazione di Guidonia aeroporto, $89 \mathrm{~m}$ s.l.m.; $42^{\circ} 00^{\prime} \mathrm{N}-12^{\circ} 44^{\prime} \mathrm{E}$ ) risulta che i mesi più freddi sono quelli di gennaio (temperatura media $7,3^{\circ} \mathrm{C}$ ) e febbraio, mentre i mesi più caldi sono quelli di luglio e agosto (temperatura media $17,5^{\circ} \mathrm{C}$ ). $\mathrm{Si}$ contano in media 30 giorni/anno con temperature uguali o inferiori a $0^{\circ} \mathrm{C}$ e 60 giorni/anno con temperatura massima uguale o superiore a $30^{\circ} \mathrm{C}$. I valori estremi di temperatura sono stati registrati nell'agosto 1998 con $+40,4^{\circ} \mathrm{C}$ e nel gennaio 1985 con $-14,0^{\circ} \mathrm{C}$. Le precipitazioni annue, 82 giorni in media, si attestano intorno a $813 \mathrm{~mm}$ con picco minimo in estate e massimo in autunno. Il mese più piovoso è novembre (precipitazioni medie 108,0 mm), quello meno piovoso è luglio (precipitazioni medie $27,7 \mathrm{~mm}$ ). Dal punto di vista fitoclimatico, l'area è inclusa nella Regione Mediterranea di Transizione a Termotipo mesomediterraneo medio o collinare inferiore - ombrotipo subumido superiore della Regione xeroterica/mesaxerica (sottoregione mesomediterranea/ipomesaxerica) con P da 810 a $940 \mathrm{~mm}$, Pest da 75 a $123 \mathrm{~mm}$, T da 14,8 a $15,6^{\circ} \mathrm{C}$ con $\mathrm{Tm}<\mathrm{a}$ $10^{\circ} \mathrm{C}$ per 3 mesi, $t$ da 2,3 a $4,0^{\circ} \mathrm{C}$ e aridità presente, di norma, a giugno, luglio e agosto (Blasi, 1994).

Il disegno della rete idrografica generale è di tipo dendritico; l'asta principale è rappresentata dal Fosso di Santa Lucia che, alla confluenza con il Fosso 
dell'Inviolata, diventa Fosso di Marco Simone, tributario di destra del Fiume Aniene e per il quale risultano tributari di sinistra Fosso Capaldo e Fosso dell'Inviolata. L'idrografia superficiale è rappresentata essenzialmente da bacini e fossi. Fosso del Cupo e Fosso dell'Inviolata sono originati da sorgenti, gli altri hanno ritmo stagionale e sono soggetti a secche estive. Si tratta di raccolte d'acqua che presentano caratteristiche di instabilità derivanti da variazioni di livello più o meno accentuate, ovvero "stagionalmente astatiche, in quanto soggette ad uno o più periodi di asciutta" (Stoch, 2005). Il $1^{\circ}$ e $4^{\circ}$ laghetto dovrebbero rientrare nella tipologia dei "pools", mentre il $2^{\circ}$ e $3^{\circ}$ nella tipologia dei "ponds" (De Meester et al., 2005). La formazione di questi invasi è recente in quanto non si osservano sul foglio I.G.M. 1:25.000, 150 IV NE Settecamini (1949) che include l'area di Tor Mastorta (E. Curti, in verbis, 28.I.2019). Tre invasi su quattro (il $3^{\circ}$ laghetto non è visibile) si osservano sul foglio I.G.M. 1:25.000, 375 sez. IV Tìvoli (2000) a destra della A1 che separa l'area di Tor Mastorta dalla discarica dell'Inviolata (Fig. 3).

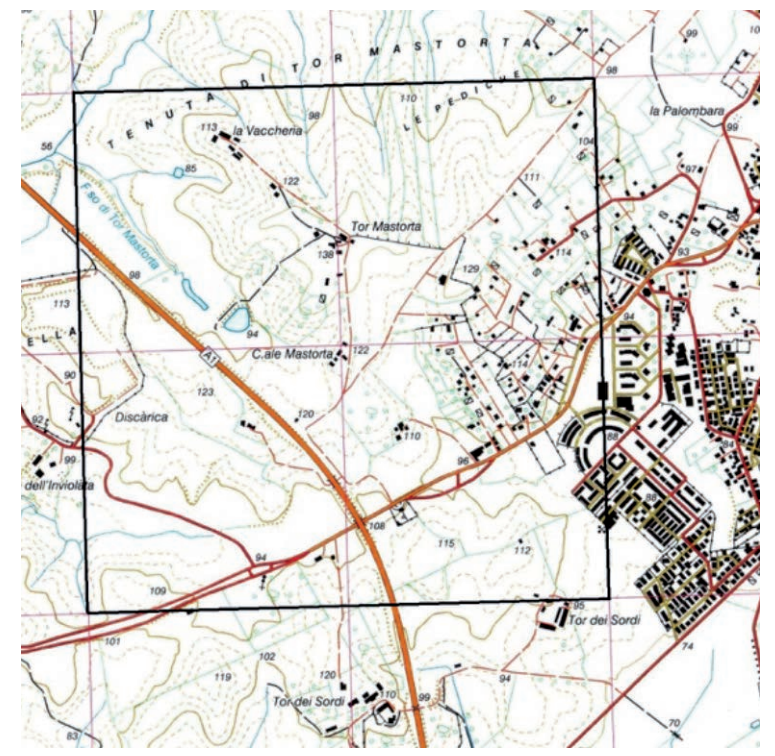

Fig. 3. Stralcio del foglio IGM 1:25.000 375 sez. IV - Tivoli (2000); nel riquadro, l'area di Tor Mastorta con i laghetti 1, 2 e 4 in alto a sinistra.

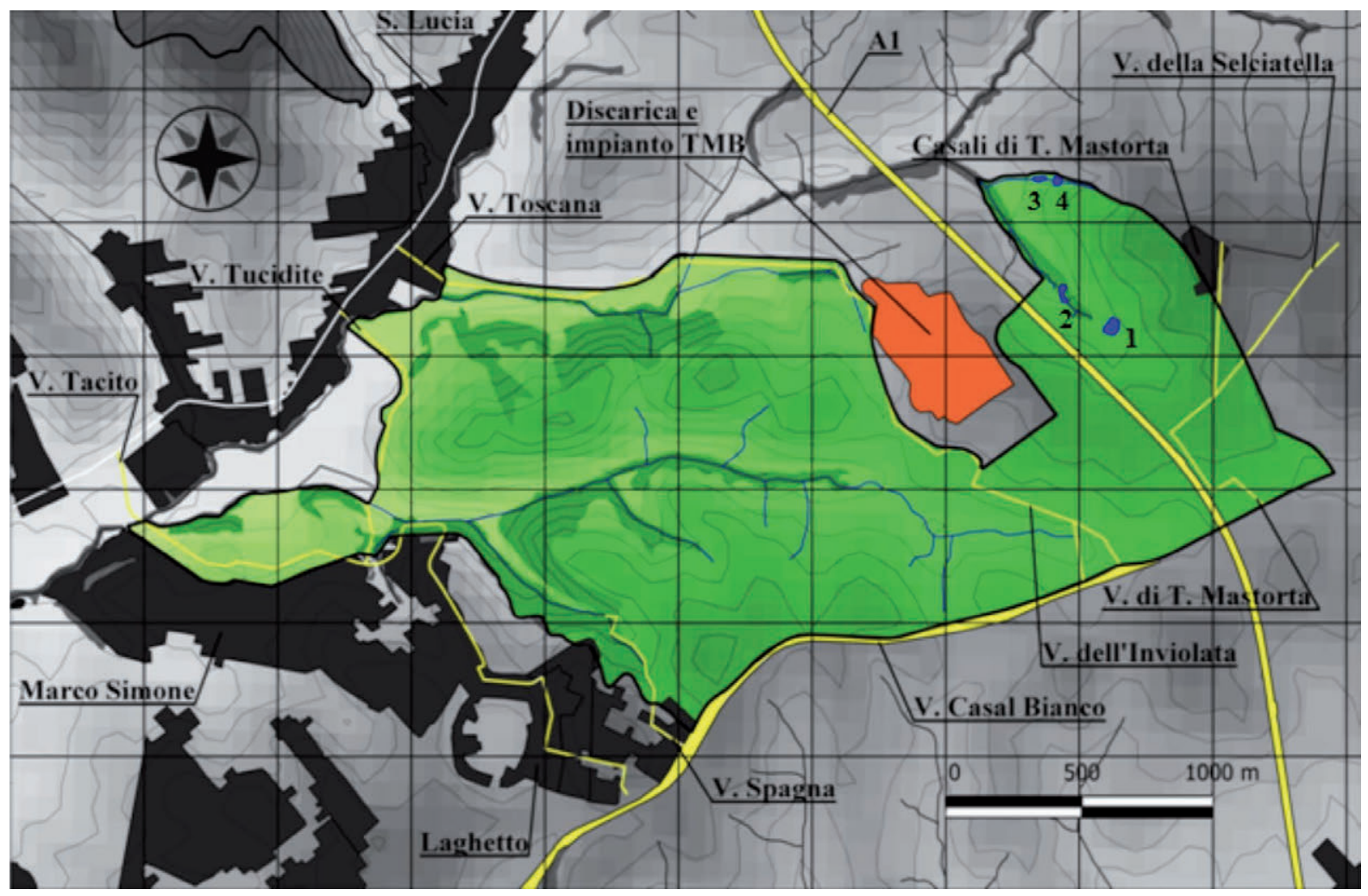

Fig. 2. Principali siti di campionamento; 1, 2, 3, 4 laghetti di Tor Mastorta. 
L'età massima ipotizzabile di almeno tre dei quattro invasi è verosimilmente di circa 70 anni. Il primo di questi laghetti (94 m s.l.m., perimetro $330 \mathrm{~m}$, superficie 7400 $\mathrm{m}^{2}$ ), scavato alla base dei rilievi circostanti e a fondo argilloso, ha forma subcircolare ed è soggetto a prosciugamento nelle estati più secche; durante le precipitazioni più intense, una parte dell'acqua deriva dal deflusso dei versanti limitrofi. Sulla sponda nord-est del laghetto è presente un canneto ad Arundo donax; ad est è circoscritto da un opus reticulatum della principale villa romana della Tenuta di Tor Mastorta (Mari, 2005). Nella sponda sud-est sono presenti Salix alba e Juncus inflexus; in acqua, Najas minor e Sporobolus schoenoides (Giardini, 2017a,b). Il secondo laghetto $(87 \mathrm{~m}$ s.l.m., perimetro $270 \mathrm{~m}$, superficie $2520 \mathrm{~m}^{2}$ ) può essere considerato un allargamento del Fosso di Tor Mastorta sul cui fondo argilloso è stato scavato l'alveo, alimen- tato sia dalle acque del fosso sia da quelle della sua modesta falda; è circondato da siepi di Rubus ulmifolius e da A. donax con Quercus robur; molto scarse le idrofite lungo le rive. I rimanenti bacini sono stati scavati dall'affluente di destra del Fosso di Tor Mastorta, da noi denominato Fosso La Vaccheria, dato che scende dall'omonimo casale e il suo nome non è riportato in cartografia (F. Stoch, in litteris, 24.VIII.2016 e 17.VII.2018). Il terzo bacino ( $82 \mathrm{~m}$ s.l.m., perimetro $165 \mathrm{~m}$, superficie $1430 \mathrm{~m}^{2}$ ), di forma subcircolare, presenta un canneto ad $A$. donax sulla sponda ovest, che, con fitte siepi di R. ulmifolius, lo separa dal quarto laghetto. Il quarto bacino $(85 \mathrm{~m}$ s.l.m., perimetro $150 \mathrm{~m}$, superficie $1600 \mathrm{~m}^{2}$ ), di forma circolare, presenta sponde accessibili con difficoltà; in giugno e luglio, si forma un'ampia fascia di vegetazione acquatica bordiera costituita principalmente da $N$. minor, Lemna gibba e Po-

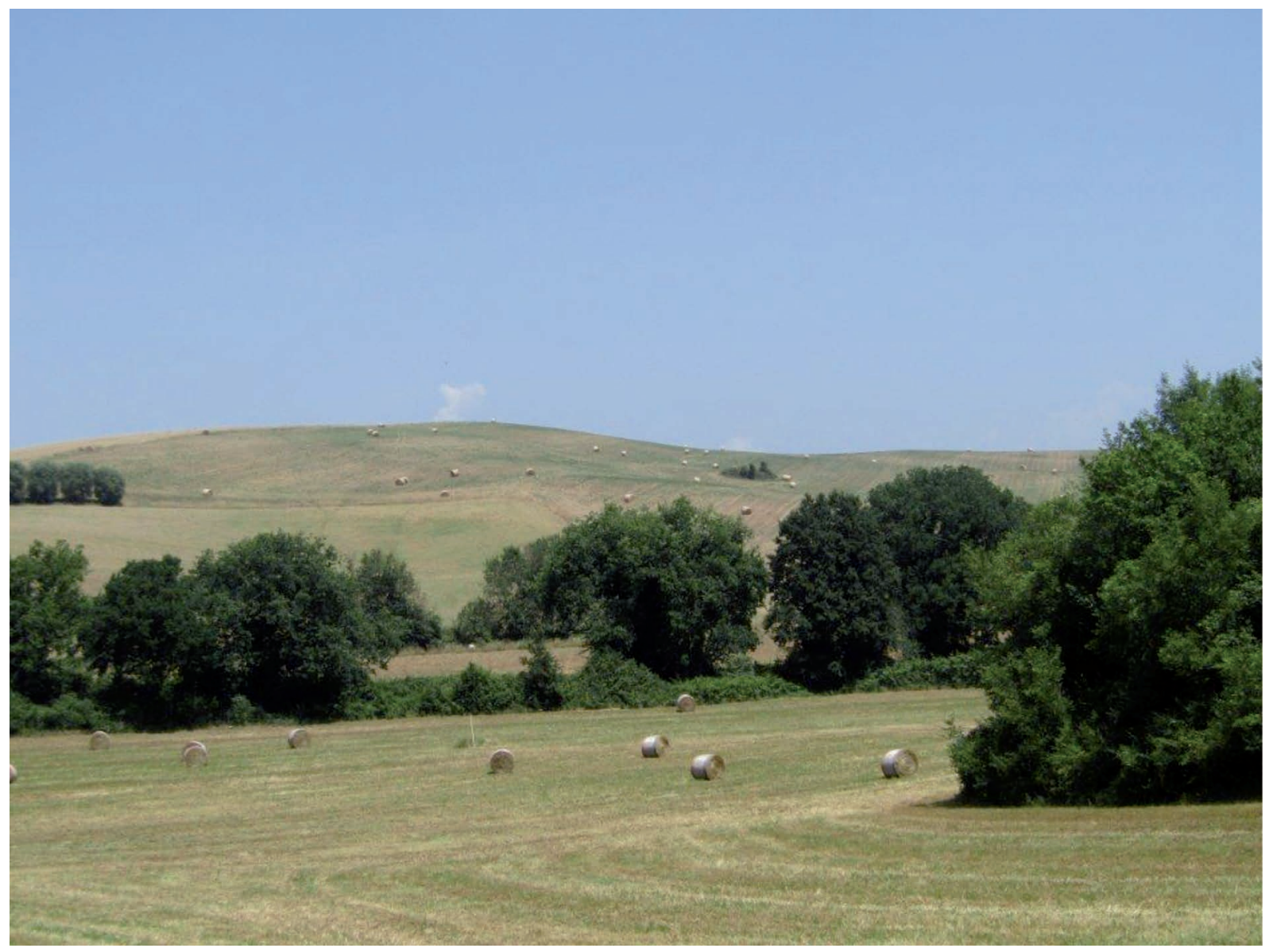

Fig. 4. Agrosistemi nel PNA Inviolata (foto di L. Tringali). 
Checklist di alcuni gruppi selezionati dell'entomofauna del Parco Naturale Archeologico dell'Inviolata

tamogeton nodosus. La maggior parte dei fossi sono ricoperti da boschi a galleria con Acer campestre, Carex pendula, Corylus avellana, Equisetum telmateja, Hedera helix, Petasites hybridus, Populus nigra, Quercus cerris, $Q$. robur, Salix alba, Sambucus ebulus, Ulmus minor (Giardini, 2005).

In funzione del grado di naturalità e della complessità strutturale è possibile riconoscere numerose tipologie vegetazionali che vanno dalla vegetazione igrofila dei fossi e dei laghetti agrari, nella quale spicca la presenza della farnia $Q$. robur, ai frammenti boschivi a dominanza di cerro $Q$. cerris, alla vegetazione rupestre delle forre tufacee, delle aree coltivate, dei pascoli e degli incolti; gran parte della superficie è interessata dalle attività agricole (frumento, erba medica, uliveti) e dall'allevamento ovino (Figg. 4-6).

Nel Parco sono state sinora censite circa 430 specie di piante vascolari appartenenti a 87 famiglie. L'unica specie di interesse comunitario è Ruscus aculeatus, incluso nell'Allegato 5 della Direttiva Habitat. Alcuni aspetti della vegetazione ripariale a salici e pioppi sono riconducibili all'habitat 92A0: Foreste a galleria di Salix alba e Populus alba. Sono incluse nell'All. B della CITES: Anacamptis pyramidalis, Ophrys apifera, Orchis purpurea, Platanthera bifolia, Serapias vomeracea, Cyclamen hederifolium e C. repandum. Imperata cilindrica, osservata da Montelucci (1941), è tutelata ai sensi della L.R. 19 settembre 1974 n. 61. Tra le specie rare o interessanti per il Lazio citiamo Najas minor e Sporobolus schoenoides rinvenute sulle sponde del primo laghetto (Giardini, 2017a,b) oltre alla brasca nodosa Potamogeton nodosus osservata nel secondo e nel quarto laghetto.

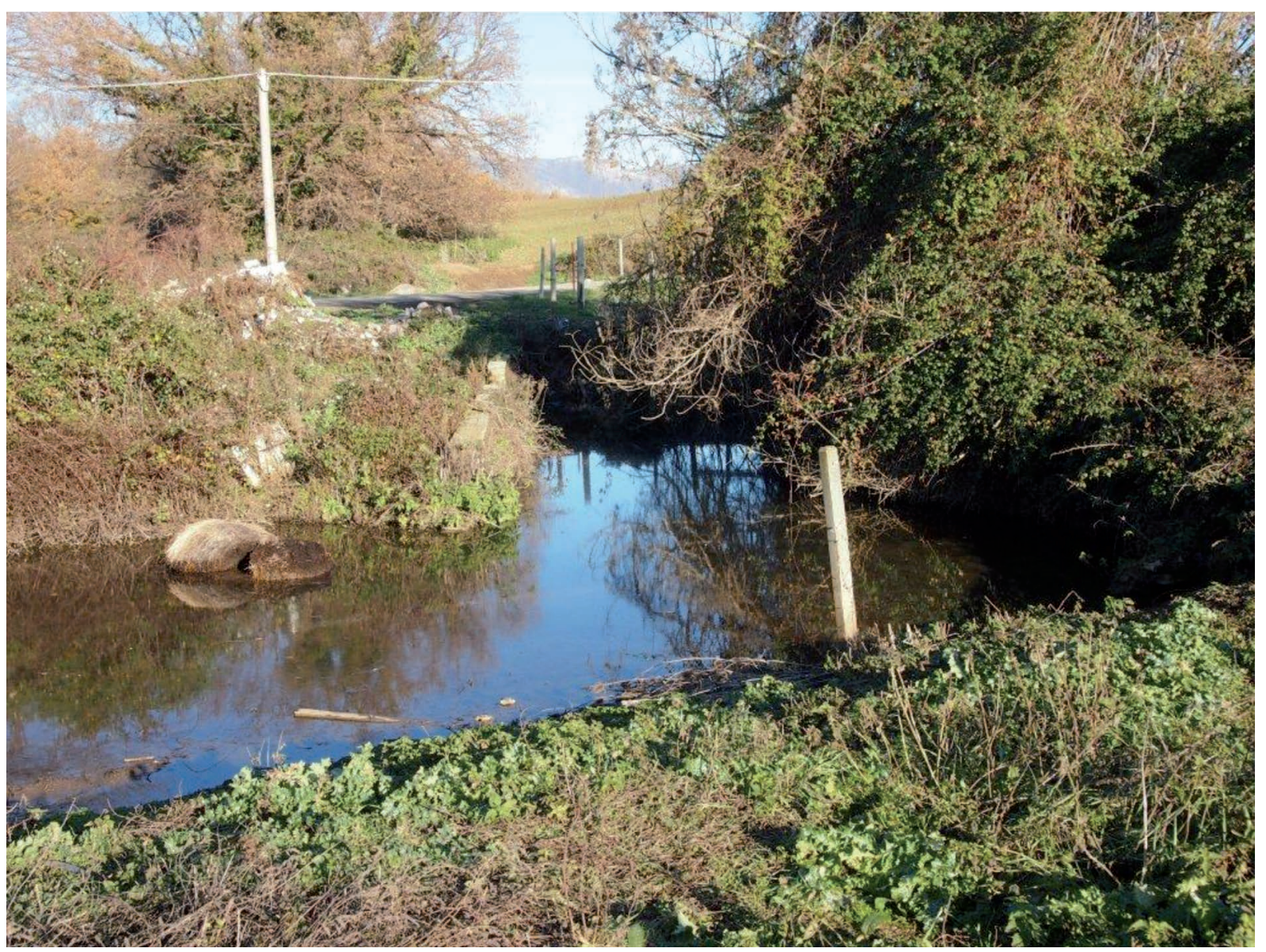

Fig. 5. Fosso del Cupo (foto di F. Cervoni). 


\section{Metodologie di indagine}

La programmazione delle ricerche è iniziata nel 2016, prevedendo una frequenza di monitoraggi sul campo pari a 1 monitoraggio/settimana; la frequenza effettiva è risultata di poco più bassa 0,8 monitoraggi/settimana. I risultati discussi derivano da monitoraggi effettuati nel periodo 02.VIII.2016 30.IV.2019 per complessivi 105 sopralluoghi in altrettante date: 18 nel 2016, 40 nel 2017, 44 nel 2018, 3 nel 2019 con numero di sopralluoghi/mese così distribuito (mesi in numeri romani); I - 3; II - 9; III - 7; IV - 10; V - 7; VI - 12; VII - 5; VIII - 15; IX - 16; X - 7; XI - 4; XII - 9; media dei sopralluoghi/mese 3,18. I tre siti di accesso all'area protetta - Via Toscana presso Santa Lucia di Fonte Nuova, Via Spagna e Via Casal Bianco presso Marco Simone, Via di Tor Mastorta e Via della Selciatella presso Guidonia, distano pochi chilometri dalla sede della SRSN; ciò ha consentito monitoraggi periodici, frequenti e prolungati. La tipologia dei sopralluoghi è così ripartita: 60 in orario antimeridiano (A), effettuati di norma tra le ore 08:00 e le13:00; 17 in orario pomeridiano (P), h 13:00 - 18:00; 11 in orario notturno $(\mathrm{N})$, h 18:00 - 24:00; 17 sopralluoghi misti (A-P, A-N, P-N, A-P-N). Il numero di rilevatori/sopralluogo è variato tra 1 e 15 con una media di 4-5/sopralluogo. Sono stati effettuati tre campi con attendamenti in loco nei periodi 13-16.IV.2017, 3031.III.2018 e 28-29.IV.2018; gli ultimi due nell'ambito, rispettivamente, delle attività del $3^{\circ}$ BioBlitz del progetto BioLazio della SRSN e del Save the Frogs Day (in collaborazione con SHI Societas Herpetologica Italica). Durante i sopralluoghi sono stati percorsi transetti prefissati che hanno consentito l'esplorazione di tutti i principali biotopi del PNA Inviolata; ambienti

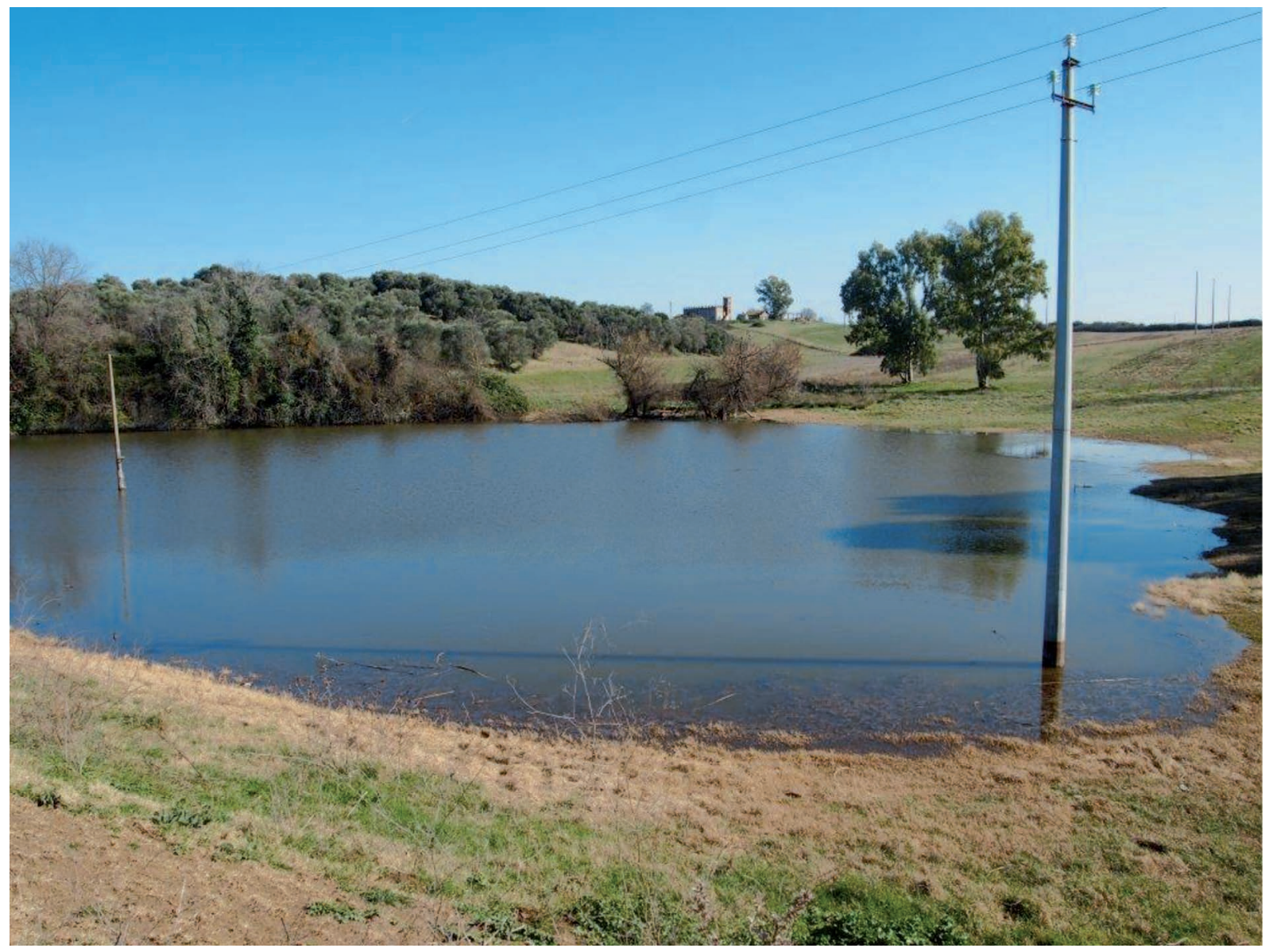

Fig. 6. $1^{\circ}$ laghetto di Tor Mastorta in condizioni di massimo invaso (foto di F. Cervoni). 
umidi (laghetti agricoli, fossi, pozzi artesiani), edifici antropici non abitati (Castello di Tor Mastorta e adiacenze, casali), ipogei e ambienti ruderali (Via Toscana, Via Spagna, Marco Simone), boschi (Via Toscana), siepi e arbusti. La presenza di terreni privati recintati ha limitato l'accesso al frammento boschivo di Via Toscana nel settore del Parco ai margini dell'abitato di Santa Lucia di Fonte Nuova.

Di seguito sono dettagliate le metodologie di studio, raccolta e determinazione dell'entomofauna (Zangheri, 2001; Brandmayr et al., 2005; Gibb \& Oseto, 2006; Groppali et al., 2008; Crucitti et al., 2015; Crucitti, 2018).

1) Campionamento diretto. Effettuato nel corso di tutti monitoraggi, è consistitito nel vaglio della lettiera, controllo delle infiorescenze e battitura del fogliame; sono stati spostati e/o sollevati pietre, cortecce, frammenti lignei, cumuli di foglie, materiali artificiali, covoni e strame di paglia (Crucitti et al., 2016). Le specie inconfondibili sono state determinate a vista o raccolte a mani nude, o con pinzette entomologiche o flaconi, oltre a retini entomologici a sacco singolo e da sfalcio. L'aspiratore è stato utilizzato per il recupero di piccoli esemplari sulle infiorescenze o all'interno del retino da sfalcio ove erano caduti.

2) Campionamento indiretto: i) otto pitfall-trap ( $p t)$ innescate e collocate il 24.IX.2017 e recuperate il 08.X.2017; sei $p t$ innescate e collocate l'11.II.2018 e recuperate il 25.II.2018; ii) dodici trappole aeree, collocate sui rami della vegetazione arborea il 12.V.2018 e ritirate il 2.VI.2018; iii) light sheet, teli bianchi illuminati per mezzo di lampade a luce bianca e/o LED UV $(\lambda=395 \mathrm{~nm})$ posizionati ai margini del bosco o nel sottobosco in ore notturne (15.IV.2017, 24.VI.2017, 12.VI.2018, 16.VI.2018, 17.VII.2018, 03.VIII.2018); iv) ricerca con faro effettuata, nel corso di tre sopralluoghi notturni, con automobile a bassa velocità e luci anabbaglianti lungo il transetto Via Toscana - Via dell'Inviolata presso Santa Lucia di Fonte Nuova (VII-VIII.2017).

3) Ispezione di resti di Vertebrati in decomposizione.

4) Ispezione delle feci di Mammiferi sia erbivori sia carnivori.

5) Ispezione della vegetazione acquatica e dei detriti sul fondale degli invasi per mezzo di retini per acquari.

Le osservazioni sono state trascritte su schede standardizzate dalle quali sono stati estrapolati i dati assolutamente certi $(\mathrm{n}>10.000)$; questi ultimi, riuniti in database, sono stati analizzati soprattutto per evidenziare trend bio-ecologici, in particolare fenologici. Le checklist da noi ottenute sono state comparate con le liste di specie note nelle aree limitrofe della Campagna Romana (Crucitti et al. 2015, 2016).

Si riporta l'elenco dei determinatori dei taxa appartenenti ai gruppi considerati. Odonata: D. Brocchieri, F. Cervoni, P. Crucitti, A. Minicò, M. Pavesi. Polyneoptera (Mantodea, Isoptera, Orthoptera, Phasmatodea, Dermaptera): M. Villani. Carabidae: M. Agosti, L. Bodei, D. Brocchieri, S. Cosimi, N. Cuffiani, A. Degiovanni, G. Franzini, P. Leo, N. Massimo, J. Matějiček, G. Pace, R. Rattu, R. Sciaky, M. Villani. Coleotteri acquatici (Dytiscidae, Gyrinidae, Haliplidae, Hydrophilidae): N. Cuffiani, L. Forbicioni, N. Massimo, A. Pinna, M. Przewoźny, A. Ruicanescu, M. Toledo, G. von Mörl, M. Pavesi. Silphidae: N. Cuffiani, M. Gigli. Staphylinidae: N. Cuffiani, P. Leo, J. Matějiček, N. Pilon, M. Villani. Cantharidae/Ptiliidae: A. Morelli. Dermestidae: G. Pezzi, V. Titov. Cleridae: I. Zappi. Aphodiidae: A. Marino, S. Ziani. Lucanidae: F. Tomasi. Scarabaeidae: G. Montanaro, S. Ziani. Geotrupidae: L. Bodei. Elateridae: S. Cosimi, G. Platia, E. Pulvirenti. Ripiphoridae / Cebrionidae: R. Rattu. Buprestidae: G. Altadonna, G. Curletti, M. Gigli, F. Izzillo, F. Rosso, A. Verdugo. Drilidae: F. Fanti, R. Rattu. Lampyridae: E. Pulvirenti. Cryptophagidae / Erotylidae / Laemophloeidae / Silvanidae / Zopheridae: D. Maccapani. Dasytidae / Malachiidae: G. Franzini. Coccinellidae: S. Cosimi, G. Mariani. Anthicidae: S. Cosimi, A. Degiovanni, P. Leo, R. Rattu. Nitidulidae: P. A. Audisio. Meloidae: M. A. Bologna. Tenebrionidae: G. Altadonna, T. Gazurek, G. Giovagnoli, F. Izzillo, P. Leo, A. Marata, G. Pezzi, R. Rattu. Cerambycidae: M. Gigli, G. Giovagnoli, F. Izzillo, A. Pauls, A. Pennisi, R. Poloni, R. Rattu, V. Viglioglia, M. Villani. Chrysomelidae: E. Berrilli, M. Bollino, L. Borowiec, G. Franzini, M. Gigli, L. Innocenti, A. Pauls, G. Pezzi. Curculionidae: S. Biondi, R. Casalini, E. Colonnelli, L. Forbicioni, E. Gatti, E. Obidin, A. Paladini, L. Pancini, A. Rattu, R. Rattu, I. Zabaluev. Rhynchitidae: S. Biondi. Apionidae: P. Kresl. Lepidoptera: V. Viglioglia.

\section{Risultati E Discussione}

Sono stati censiti 533 taxa di 101 famiglie; 510 a livello di specie, 23 a livello di genere (17 Coleoptera, 5 Lepidoptera, 1 Mecoptera). L'ordine maggior- 
mente rappresentato è quello dei Coleoptera (359 taxa di cui 343 determinati a livello di specie) con i Carabidae (77 taxa, 76 specie) come famiglia più rappresentata; seguono i Lepidoptera (107 taxa, 102 specie) (Appendice: Tabb. A1-5). L'ordine delle famiglie segue la sistematica adottata nella Checklist of the Italian fauna (Stoch, 2003) mentre, nell'ambito di ciascuna famiglia, generi e specie sono elencati in ordine alfabetico.

\section{Odonata}

Sono state censite 28 specie su 42 citate della città di Roma delimitata dal GRA (Utzeri \& Dell'Anna, 1997), 58 del Lazio e 93 d'Italia (Riservato et al., 2014) $(66,6 \%, 48,2 \%$ e $30,1 \%$, rispettivamente) incluse in 18 generi di 7 famiglie. Tredici specie (11 generi di 4 famiglie) sono Zygoptera, 15 specie (8 generi di 3 famiglie) sono Anisoptera. Gli Odonati dell'Inviolata sono stati oggetto di uno specifico contributo (Brocchieri et al., 2018), rispetto al quale è stata aggiunta Sympecma fusca, osservata nell'aprile 2019 presso il $1^{\circ}$ laghetto di Tor Mastorta (Fig. 7). Pertanto il totale delle specie censite nella Campagna Romana a nord-est di Roma ammonta a 35, pari al 37,6\% del totale nazionale e al $60,3 \%$ delle specie note per il Lazio (Brocchieri et al., 2014; Crucitti et al., 2016; Brocchieri et al., 2018). L'analisi dei corotipi permette di constatare la netta dominanza di elementi ad ampia distribuzione nella regione Olartica (20 specie, $71,4 \%)$. Nettamente inferiori i contingenti a gravitazione europea ( 3 specie, $10,7 \%$ ), a gravitazione medi-

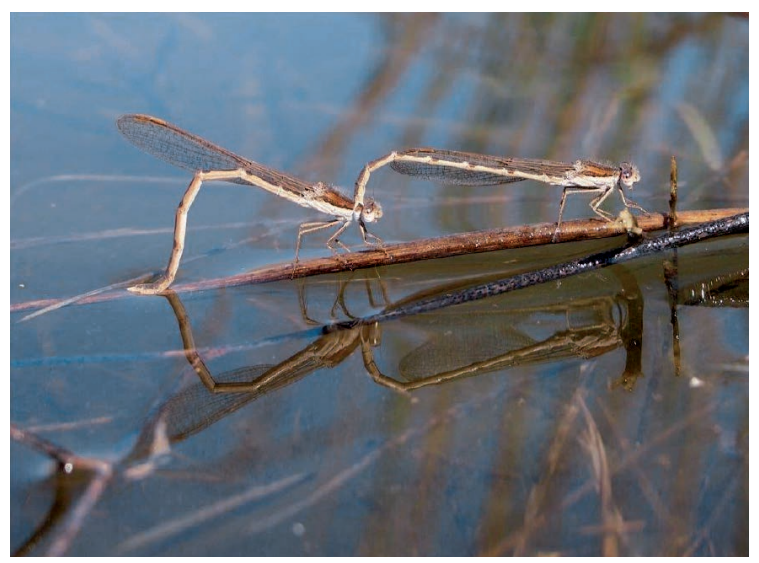

Fig. 7. Sympecma fusca in tandem e ovideposisizione (foto di F. Cervoni). terranea ( 2 specie, $7,2 \%$ ) e di origine afrotropicale (3 specie, $10,7 \%)$.

\section{Mantodea, Isoptera, Orthoptera, Phasmatodea, Dermaptera}

I Mantodea sono rappresentati da 5 specie di 2 famiglie. Si segnala la presenza di Ameles spallanzania, sinora non campionata nella Campagna Romana a nord-est di Roma (Crucitti et al. 2015, 2016). Nel caso degli Isoptera, l'area del PNA Inviolata deve essere aggiunta all'elenco dei siti termitici per entrambe le specie italiane (Sbrenna \& Micciarelli Sbrenna, 2008). Gli Orthoptera ammontano a 25 specie, numero inferiore al reale popolamento ortotterologico dell'area, risultato imputabile a campionamenti non strettamente finalizzati. Si segnala la presenza di Dolichopoda geniculata geniculata, endemismo appenninico abbondante nel sito dell'Ipogeo di Monte dell'Incastro (cavità artificiale) e di Mogoplistes brunneus, considerato poco frequente nell'Italia centro meridionale (Massa et al. 2012). Tra i Dermaptera, Labia minor viene citata per la prima volta nella Campagna Romana; la presenza di questa specie è favorita dalla permanenza di ambienti umidi in buone condizioni.

\section{Coleoptera Carabidae}

Sono rappresentati da 77 taxa di cui 76 specie, il 13,5\% delle specie del Lazio (560; A. Vigna Taglianti, in verbis, I.2013). Rispetto ai lavori di Crucitti et al. (2015, 2016), 32 specie non sono citate per la Campagna Romana a nord-est di Roma; con il presente lavoro il numero di specie note per le aree sinora studiate (Nomentum, Gattaceca-Barco e aree limitrofe, Marco Simone, PNA Inviolata) ammonta a 109, il $19,5 \%$ delle specie del Lazio, valore che offre un quadro sufficientemente completo della carabidofauna dell'area. La maggior parte delle specie è comune. $\mathrm{Ca}$ rabus coriaceus è stato campionato in una sola occasione lungo Via dell'Inviolata nel corso di una ricerca notturna con faro (VIII.2017). Poecilus cursorius, campionato in una sola occasione (VIII.2018), è specie un tempo discretamente diffusa negli ambienti umidi d'Italia, divenuta più rara in seguito alle opere di bonifica (Pesarini \& Monzini, 2011). Epomis circumscriptus, considerata ormai molto rara in quanto scomparsa da numerosi siti a causa della progressiva distruzione dell'habitat (Pesarini \& Monzini, 2011), è stata rinvenuta esclusivamente nel sito del $1^{\circ}$ laghetto 
di Tor Mastorta, ove è presente una popolazione relativamente abbondante (2-10 exx./singola visita) sebbene confinata in un'area palustre di poche centinaia di $\mathrm{m}^{2}$ compresa tra l'opus reticulatum della villa romana e il boschetto di salici limitrofo al bacino. Le larve di E. circumscriptus (e quelle dell'affine Epomis

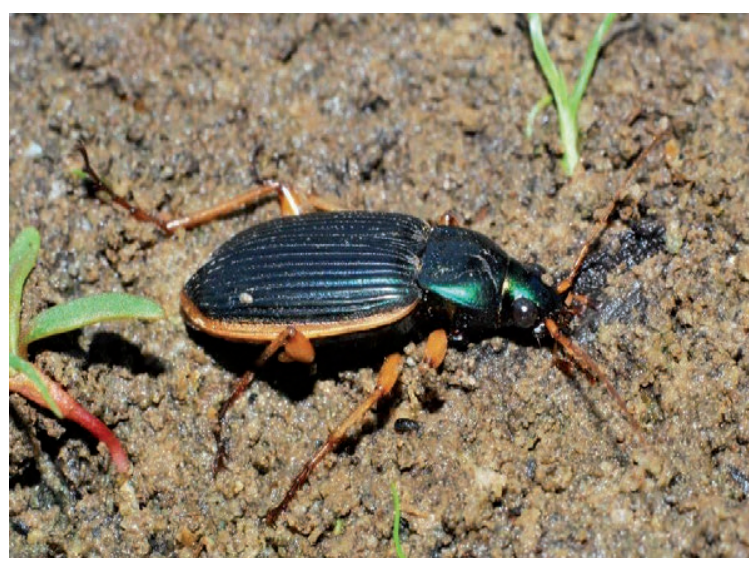

Fig. 8. Adulto di Epomis circumscriptus (foto di L. Tringali). dejeani Dejean, 1831) risultano predatrici di neometamorfosati di Anura Bufonidae, effetto noto come prey-predator reversal (Wizen \& Gasith, 2011); nel contesto, si rileva la presenza, nel $1^{\circ}$ laghetto, di popolazioni numerose di Bufo bufo (Linnaeus, 1758) e di Bufotes balearicus (Boettger, 1880) (Cervoni et al., 2018) (Fig. 8).

La maggiore abbondanza di dati sui Carabidae dell'area ha suggerito l'analisi dei corotipi, la quale ha evidenziato la predominanza di elementi ad ampia distribuzione (46 specie, 59,7\%), il sostanziale equilibrio tra elementi europei ( 15 specie, $19,5 \%$ ) e mediterranei (12 specie, 15,6\%) nonchè il minore contributo del contingente di taxa a distribuzione paleotropicale-mediterranea (3 specie, 3,9\%); valori in linea con i risultati ottenuti per i comprensori dell'area arcipelago mentanese-cornicolano precedentemente indagati (Crucitti et al., 2015) (Tab. 1). Quattro specie (5,2\%) sono endemiche appenniniche (Appendice: Tab. A6).

\section{Coleoptera (Carabidae esclusi)}

Sono stati censiti 282 taxa di 54 famiglie, 246

Tab.1. Carabidae: numero di specie per categoria corologica e valori percentuali rispetto al totale.

\begin{tabular}{|c|c|c|}
\hline Corotipo & Specie & $\%$ \\
\hline A - SPECIE A DISTRIBUZIONE OLARTICA & 46 & 60,5 \\
\hline Paleartica & 10 & 13,1 \\
\hline Paleartica-Occidentale & 2 & 2,6 \\
\hline Asiatico-Europea & 5 & 6,5 \\
\hline Sibirico-Europea & 1 & 1,3 \\
\hline Centroasiatico-Europeo-Mediterranea & 2 & 2,6 \\
\hline Turanico-Europeo-Mediterranea & 4 & 5,2 \\
\hline Turanico-Europea & 9 & 11,8 \\
\hline Europeo-Mediterranea & 13 & 17,1 \\
\hline B - SPECIE A DISTRIBUZIONE EUROPEA & 15 & 19,7 \\
\hline Europea $s$. str. & 7 & 13,1 \\
\hline S-Europea & 8 & 10,5 \\
\hline C - SPECIE A DISTRIBUZIONE MEDITERRANEA & 12 & 15,7 \\
\hline Mediterranea s. str. & 7 & 9,2 \\
\hline Mediterranea-Occidentale & 4 & 5,2 \\
\hline Mediterranea-Orientale & 1 & 1,3 \\
\hline D - SPECIE A DISTRIBUZIONE PALEOTROPICALE-MEDITERRANEA & 3 & 3,9 \\
\hline Afrotropicale-Paleartico & 1 & 1,3 \\
\hline Afrotropicale-Mediterranea & 1 & 1,3 \\
\hline Afrotropicale-Indiano-Mediterraneo & 1 & 1,3 \\
\hline TOTALE & 76 & 100 \\
\hline
\end{tabular}


dei quali determinati a livello specifico, 17 determinati a livello di genere e 20 in forma dubitativa. La presenza di fossi e canali caratterizzati da acque in buone condizioni trofiche ha permesso il campionamento di alcune specie di Coleotteri acquatici delle famiglie Haliplidae, Dytiscidae, Gyrinidae e Hydrophilidae per le quali esistono pochi dati bibliografici relativi alla Campagna Romana a nord est di Roma. Gli Staphylinidae sono rappresentati da 16 specie, tipiche delle formazioni boschive collinari a latifoglie termofile, spesso legate a materiale in decomposizione, di origine sia animale sia vegetale (Zanetti \& Tagliapietra, 2004); si segnala l'endemismo appenninico Ocypus italicus. Complessivamente, sono state raccolte 39 specie di Scarabaeoidea le quali, in funzione del regime trofico, possono essere così ripartite: 2 cheratinofaghe ( 2 Trogidae), 23 coprofaghe (1 Geotrupidae, 13 Aphodiidae, 9 Scarabaeidae), 14 fitofaghe (2 Lucanidae, 1 Glaphyridae, 2 Melolonthidae, 2 Dynastidae, 7 Cetoniidae). Le specie coprofaghe abbondano nelle aree di Tor Mastorta e della Torraccia dell'Inviolata, intensamente sfruttate per il pascolo ovino; nei siti di stazionamento delle greggi sono stati raccolti i rappresentanti della famiglia Trogidae; le specie fitofaghe sono state raccolte in corrispondenza dei filari boscosi che bordano i fossi, sia a vista sia mediante trappole aeree e light sheet. Gli Elateridae sono rappresentati da 17 specie, tutte più o meno comuni. I Buprestidae sono rappresentati da 20 specie, in prevalenza dei generi Agrilus e Anthaxia: merita di essere menzionata $A$. lucens, nuova per il Lazio, regione che quindi raggiunge il totale di 115 specie di Buprestidae di cui 24 nel solo genere Anthaxia (Curletti et al.,
2003). Tra i Tenebrionidae (16 specie) si segnala l'endemismo Colpotus strigosus strigosus e le specie silvicole Platydema violacea e Uloma culinaris, considerate rare e legate ad aree in buone condizioni di conservazione (Fattorini, 2013). Cerambycidae e Chrysomelidae sono rappresentati da 30 e 26 specie rispettivamente, $i$ primi in gran parte floricoli e campionati a vista su infiorescenze, i secondi con il retino da sfalcio. Anche all'Inviolata, come in altre aree della Campagna Romana, sono presenti le specie alloctone di origine orientale Xylotrechus stebbingi e Gonioctena fornicata; al contrario, non sono stati riscontrati Cerambycidae del genere Phoracantha nonostante la presenza di numerosi eucalipti. Le specie di Curculionoidea ammontano a 33; tra queste si citano il punteruolo rosso delle palme Rhyncophorus ferrugineus, alloctono ormai ubiquista nella Campagna Romana, e il tarlo del fico o punteruolo nero del fico Aclees sp., altro taxon invasivo già noto per le aree limitrofe di Marco Simone e Setteville Nord (Crucitti et al., 2015, 2016); infine, le specie endemiche appenniniche Otiorhynchus armatus e Phyllobius etruscus. Sono raffrontati i dati dei taxa (famiglie, superfamiglie) di Coleoptera maggiormente rappresentati per numero di specie, con quelli di altre aree della Campagna Romana (Tab. 2). Complessivamente, 7 specie sono endemiche (Appendice: Tab. A7).

\section{Lepidoptera, Mecoptera}

Anche tra i Lepidoptera si rileva una notevole dispersione dei taxa campionati, pari a 107, ripartiti tra 89 generi di 21 famiglie, considerando i Satyrinae sottofamiglia di Nymphalidae, Arctiinae e Lymatriinae

Tab. 2. Comparazione tra PNA Inviolata e altre aree della Campagna Romana a nord-est di Roma a livello dei taxa (famiglie, superfamiglie) maggiormente rappresentati per numero di specie.

\begin{tabular}{lccc}
\hline Taxa & $\begin{array}{c}N^{\circ} \text { specie } \\
\text { del PNA Inviolata }\end{array}$ & $\begin{array}{c}N^{\circ} \text { di specie diverse note in altre aree } \\
\text { della Campagna Romana } \\
\text { (Crucitti } \text { et al., 2015, 2016) }\end{array}$ & $\begin{array}{c}\text { Totale specie } \\
\text { della Campagna Romana }\end{array}$ \\
\hline Carabidae & 77 & 32 & 109 \\
Staphylinidae & 16 & 4 & 20 \\
Scarabaeoidea & 39 & 15 & 54 \\
Elateridae & 17 & 4 & 21 \\
Buprestidae & 20 & 6 & 26 \\
Tenebrionidae & 16 & 5 & 21 \\
Cerambycidae & 30 & 19 & 49 \\
Chrysomelidae & 26 & 7 & 33 \\
Curculionoidea & 33 & 16 & 49 \\
\hline
\end{tabular}


sottofamiglie di Erebidae (Mazzei et al., 2019). L'esiguo numero di Microheterocera è imputabile alle tecniche non specializzate di raccolta di un gruppo peraltro di non facile campionamento. I Macroheterocera sono i più numerosi, con 61 specie; tra queste le più interessanti sono il Saturnidae Saturnia pavoniella, segnalata per i vicini Monti Cornicolani (Giardini, 2012) ma finora mai campionata nella Campagna Romana a nord-est di Roma, al contrario della congenere S. pyri (Crucitti et al., 2015) (Fig. 9). Il Lasiocampide Eriogaster catax risulta inserita negli elenchi delle specie tutelate dalla Direttiva Habitat (92/43/CEE) (D’Antoni et al., 2003). Molti rappresentanti delle famiglie Erebidae e Noctuidae sono state compionate con la tecnica del light sheet ai margini o all'interno dei boschi mischi di latifoglie; altre specie sono state trovate in alcune cavità naturali o seminaturali, come

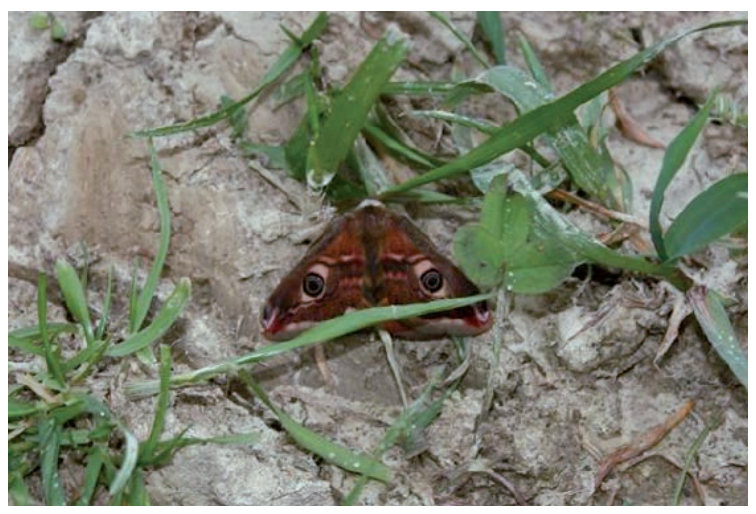

Fig. 9. Saturnia pavoniella (foto E. Di Russo).
Euplagia quadripunctaria, inserita anch'essa in Direttiva Habitat (D'Antoni et al., 2003). Le 36 specie di Ropaloceri offrono un quadro abbastanza esauriente del popolamento lepidotterologico dei cosiddetti "Diurna"; trattandosi in gran parte di specie appariscenti, dai colori vivaci e attive nelle ore centrali della giornata, si prestano bene ad un campionamento anche non specializzato. In relazione alle caratteristiche dell'area di studio, Papilionidae e Nymphalidae sono adeguatamente rappresentati al contrario dei Pieridae e soprattutto dei Lycaenidae che potrebbero riservare ulteriori sorprese con il proseguimento delle ricerche. Il numero di specie ripartite secondo la divisione classica dell'ordine Lepidoptera nei gruppi Microheterocera, Macroheterocera e Rhopalocera è sintetizzato in Tab. 3.

\section{CONCLUSIONI}

Il Parco dell'Inviolata risente, analogamente ad altre aree di interesse naturalistico della Campagna Romana a nord-est di Roma, della condizione comune a molti territori a mosaico; piccoli comprensori protetti inclusi in una matrice costituita da una estesa rete di infrastrutture viarie e da edificato sparso/compatto a carattere abitativo residenziale. La sua gestione, affidata per 20 anni al Comune di Guidonia Montecelio, tra i più estesi e ad alta densità abitativa della Provincia di Roma, ha esposto quest'area alle mire della speculazione edilizia e all'abnorme e illecito ampliamento dell'omonima discarica per rifiuti solidi urbani, seconda, nel Lazio, solo a quella di Malagrotta (CRA Comitato per il Risanamento Ambientale, 2016). Ulteriori interventi lesivi dell'integrità del Parco risultano

Tab. 3. Percentuali relative al numero di famiglie e numero di specie in Micro e Macroheterocera e in Rhopalocera.

\begin{tabular}{|c|c|c|}
\hline Taxa & $\mathrm{N}^{\circ}$ famiglie/specie & $\%$ \\
\hline \multicolumn{3}{|l|}{ MICROHETEROCERA } \\
\hline $\begin{array}{l}\text { Elachistidae, Oecophoridae, Cossidae, Sesiidae, Zygaenidae, Tortricidae, } \\
\text { Pyralidae, Crambidae, Thyrididae }\end{array}$ & $9 / 10$ & $42,8 / 9,4$ \\
\hline \multicolumn{3}{|l|}{ MACROHETEROCERA } \\
\hline $\begin{array}{l}\text { Lasiocampidae, Sphingidae, Saturniidae, Geometridae, Notodontidae, Erebidae, } \\
\text { Noctuidae }\end{array}$ & $7 / 61$ & $33,3 / 57$ \\
\hline \multicolumn{3}{|l|}{ RHOPALOCERA } \\
\hline Hesperiidae, Papilionidae, Pieridae, Lycaenidae, Nymphalidae & $5 / 36$ & $23,9 / 33,6$ \\
\hline Totale & $21 / 107$ & 100 \\
\hline
\end{tabular}


la bretella Fiano-S. Cesareo, alla quale si è recentemente aggiunto il limitrofo casello autostradale, oltre alla presenza di tutti i fenomeni di degrado tipici dei centri abitati dell'area romana a nord-est di Roma (Calamita (a cura di), 2005; Crucitti, 2013).

Malgrado ciò, si sono conservati, nel territorio dell'Inviolata, i principali caratteri paesaggistici, naturali e culturali, propri della Campagna Romana. Il fatto che l'area, da sempre a vocazione agro-silvo-pastorale, sia in gran parte occupata da estesi agrosistemi privati ha contribuito al mantenimento di un discreto grado complessivo di naturalità. Questa ricerca ha infatti permesso di accertare la presenza di numerosi elementi di interesse biogeografico ed ecologico, dato sorprendente se raffrontato alla posizione e alla modesta estensione dell'area. Il confronto tra il numero di specie di gruppi selezionati di Insecta (soprattutto Odonata, Coleoptera Carabidae e Lepidoptera "Diurna") riscontrati nel PNA Inviolata e nell'area più estesa, "arcipelago mentanese - cornicolano" (Nomentum, Gattaceca - Barco e aree limitrofe; Crucitti et al., 2013), consente di rilevare la ricchezza di specie del PNA Inviolata, imputabile soprattutto all'esistenza di numerosi biotopi umidi, di natura ed estensione diversa. È plausibile ritenere che i risultati ottenuti, in termini di numero di specie, siano prossimi alla realtà nel caso di Odonata, Mantodea, Coleoptera Carabidae e Lepidoptera "Diurna"; Orthoptera e Lepidoptera Micro e Macroheterocera richiederanno invece studi dedicati, inclusa l'adozione di metodi specifici di raccolta. Questa ricchezza di specie può essere imputabile sia alla presenza di habitat idonei sia all'effetto "concentrazione" dovuto all'intensa pressione antropica sulle popolazioni animali residenti, in particolare nelle aree limitrofe al parco. L'importanza del mantenimento di patches costituite da piccole aree boscate, agrosistemi ad elevata diversità floristica, ambienti ipogei e ruderali e aree umide è paradigmatica. Si tratta peraltro di un complesso di habitat dall'incerto futuro, in particolare i laghetti di Tor Mastorta, biotopi di elevatissimo interesse naturalistico e conservazionistico, sovrastati dalla megadiscarica dell'Inviolata. Per molteplici fattori, il sistema delle acque basse stagnanti di piccole dimensioni merita una attenzione non inferiore a quella riservata ai sistemi a larga scala, costieri e delle acque interne; non può essere infatti sottovalutata la loro importanza come modelli utili per affrontare problemi generali di ecologia e biologia evoluzionistica (De Meester et al., 2005).

\section{RINGRAZIAMENTI}

Si ringraziano numerosi soci della Società Romana di Scienze Naturali per la collaborazione nel corso delle ricerche sul campo e bibliografiche; Francesca Bombarda, Angelina Chinè, Ezio Curti, Claudio Pulvirenti, Giuliano e Leonardo Santoboni, Fabio Stoch, Luca Tringali. Siamo riconoscenti nei confronti dei numerosi specialisti che hanno collaborato alla determinazione di taxa problematici. Il Parco Naturale Regionale dei Monti Lucretili ha garantito lo svolgimento delle ricerche della SRSN a partire dalla firma del Protocollo d'Intesa tra l'Ente Parco e la Società Romana di Scienze Naturali (XI.2016). Infine, un particolare riconoscimento è dovuto ai gestori delle tenute del Parco che ne hanno consentito l'accesso in numerose occasioni.

\section{BiBLIOGRAFIA}

Blasi C., 1994 - Fitoclimatologia del Lazio. Università "La Sapienza" Roma, Dipartimento di Biologia Vegetale e Regione Lazio Assessorato Agricoltura, Foreste Caccia Pesca, Usi Civici. Tipografia Borgia, Roma.

Brandmayr P., Zetto T., Pizzolotto R., Casale A., Vigna Taglianti A., 2005 - I Coleotteri Carabidi per la valutazione ambientale e la conservazione della biodiversità. Manuale operativo. APAT, Agenzia per la protezione dell'ambiente e per i servizi tecnici, Manuali e Linee Guida. 34/2005. I.G.E.R. srl, Roma, 240 pp.

Brocchieri D., Castelluccio P., Crucitti P., 2014 - Gli Odonati della Riserva Naturale "Macchia di Gattaceca e Macchia del Barco" (Lazio) (Odonata). Bollettino della Società Entomologica Italiana, 146: 31-40.

Brocchieri D., Cervoni F., Pulvirenti E., Crucitti P., 2018 - Gli Odonati del Parco Naturale Archeologico dell'Inviolata (Guidonia Montecelio, Roma) (Odonata). Bollettino dell'Associazione Romana di Entomologia, 73(1-4): 5-21.

Bunone E., 2005 - Parco regionale dell'Inviolata: inquadramento geomorfologico. In: Calamita U. (a cura di), Atti del Convegno di Studi "Parco archeologico naturale dell'Inviolata di Guidonia, le ragioni di una tutela", Guidonia, 25 gennaio 2003.

CALAmita U. (a cura di), 2005 - Atti del Convegno di Studi "Parco archeologico naturale dell'Inviolata di Guidonia, le ragioni di una tutela", Guidonia, 25 gennaio 2003. 
Checklist di alcuni gruppi selezionati dell'entomofauna del Parco Naturale Archeologico dell'Inviolata

Calamita U. (a cura di), 2012 - Materiali propedeutici al piano d'assetto del Parco Regionale Naturale Archeologico dell'Inviolata di Guidonia. Tesi di laurea in Urbanistica e Sistemi Informativi Territoriali di Simone Quintavalle, Università "La Sapienza” Roma, anno accademico 2009-10. Con un'appendice su Scavi e Scoperte nella Tenuta dell'Inviolata di Filippo Avilia. Associazione culturale onlus “Amici dell’Inviolata”. Grafica Ripoli, Tivoli (RM).

Cervoni F., Brocchieri D., Crucitti P., Grispigni Manetti C., Marini D., Pulvirenti E., Santoboni L., 2018 - Prospetto della fauna del Parco Regionale Archeologico Naturale dell'Inviolata di Guidonia (Roma). Associazione Nomentana di Storia e Archeologia onlus, Annali 2017-2018, Nuova serie n. 17: 96-101.

CRA-Comitato per il Risanamento Ambientale, 2016 - Dossier Regione Lazio vs Inviolata Guidonia, stampato in proprio.

CRUCitTi P., 2013 - L’Arcipelago Mentanese-Cornicolano. Paesaggi frammentati della Campagna Romana. Bollettino della Società Geografica Italiana, Serie XIII, vol. VI (2013): 239-263.

CRUCITTI P., 2018 - Principi e metodi della ricerca faunistica - La progettazione nelle ricerche sulla biodiversità animale. Edizioni Accademiche Italiane, $316 \mathrm{pp}$.

Crucitti P., Amori G., Battisti C., Giardini M., 2013 - Check-list degli Anfibi, Rettili, Uccelli e Mammiferi dell'area “arcipelago mentanese - cornicolano" (Campagna Romana, Lazio). Bollettino del Museo Civico di Storia Naturale di Verona, 37 (Botanica Zoologia): 29-46.

Crucitti P., Brocchieri D., Bubbico F., Castelluccio P., Emiliani F., Francioni G., Tringali L., 2015 - Check-list di gruppi selezionati dell'entomofauna dell'area “Arcipelago Mentanese - cornicolano” (Lazio). Bollettino Società Entomologica Italiana, 147: 3-29.

Crucitti P., Bombarda F., Brocchieri D., Bubbico F., Del Bove E., Doglio S., Emiliani F., Francioni G., Gnecchi M., MiChelangeli F., Rodomontini R., Pulvirenti E., Rossi S., Santoboni L., Tringali L., Viglioglia V., 2016 - Checklist di gruppi selezionati dell'entomofauna di una area della campagna romana a nord-est di Roma (Lazio) (Insecta). Bollettino Associazione Romana di Entomologia, 71: 207-233.

Crucitti P., Giardini M., Tringali L., 2017 - Parco dell’Inviolata. Una preziosa perla della Campagna Romana. In: Parchilazio.it n.15, 23 febbraio 2017, pp. 12-13.

Curletti G., Rastelli M., Rastelli S, Tassi F., 2003 - Coleotteri Buprestidi d'Italia CD-ROM. Museo Civico di Storia Naturale di Carmagnola (Torino), Progetto Biodiversità (Roma).

D’Antoni S., Dupré E., La Posta S., Verucci P., 2003 - Fauna italiana inclusa nella Direttiva Habitat. Ministero dell'Ambiente e della Tutela del Territorio - DPN Direzione per la Protezione della Natura (Revisione Scientifica: Unione Zoologica Italiana). La Fenice Grafica - Roma.

De Meester L., Declerk S., Stoks R., Louette G., Van De Meutter F., De Bie T., Michels E., Brendonck L., 2005 - Ponds and pools as model systems in conservation biology, ecology and evolutionary biology. Aquatic Conserv: Mar. Freshw. Ecosyst., 15: 715-725.

FATTORINI S., 2013 - I Coleotteri Tenebrionidi di Roma (Coleoptera, Tenebrionidae). Fragmenta Entomologica, 45: 87-142.

FrAnK B., LoREnZETti E., 2005 - Il ruolo degli enti locali nello studio della frammentazione ambientale: esperienze nella Provincia di Roma. Atti del Convegno Nazionale "Ecoregioni e Reti Ecologiche: la pianificazione incontra la conservazione”. Roma, 27-28 maggio 2004. Provincia di Roma, Assessorato alle Politiche dell'Agricoltura, dell'Ambiente e della Protezione Civile, Unione Provincie Italiane (UPI), WWF Italia - Onlus. Edicomprint, Roma: 91-94.

Giardini M., 2005 - Cenni sull'ambiente naturale del Parco archeologico - naturale dell'Inviolata e delle tenute storiche di Guidonia-Montecelio. In: CALAMITA, U. (a cura di), Atti del Convegno di Studi “Parco archeologico naturale dell'Inviolata di Guidonia, le ragioni di una tutela”, Guidonia, 25 gennaio 2003, pp. 27-55. Associazione culturale onlus Amici dell'Inviolata Guidonia, Provincia di Roma.

Giardini M., 2012 - I lepidotteri sfingidi e saturnidi dei Monti Cornicolani (Italia centrale). In: Giardini M. (a cura di), 2012. Sant'Angelo Romano (Monti Cornicolani, Roma). Un territorio ricco di storia e di natura. Regione Lazio, Assessorato Ambiente e Sviluppo sostenibile, Comune di Sant'Angelo Romano. Grafica Ripoli, Tivoli. pp. 166-174.

Giardini M., 2017 - Nuove segnalazioni floristiche italiane 3(16): Najas minor All. In: Roma-MArzio F., PERUZZI L., BERNARdo L., Bartolucci F., De Ruvo B., De Ruvo A., Conti F., Giardini M., Domina G., Biondi E., Gasparri R., Casavecchia S., Matera R., Nuove segnalazioni floristiche italiane 3 (10-21). Notiziario della Società Botanica Italiana, p. 210.

Giardini M., 2017 b - Nuove segnalazioni floristiche italiane 3(21): Sporobolus schoenoides (L.) P.M. Peterson. In: RomA-MARzio F., Peruzzi L., Bernardo L., Bartolucci F., De Ruvo B., De Ruvo A., Conti F., Giardini M., Domina G., Biondi 
E., Gasparri R., CASAVecchia S., Matera R., Nuove segnalazioni floristiche italiane 3 (10-21). Notiziario della Società Botanica Italiana, p. 211.

GibB T.J., Oseto C.Y., 2006 - Arthropod Collection and Identification. Laboratory and Field Techniques. Elsevier Inc. USA, UK, $311 \mathrm{pp}$.

Groppali R., D’Amico G., Riccardi C., 2008 - Osservare gli insetti, farfalle e libellule del Parco Adda Sud. Atlante - guida per la fruizione della fauna minore nell'area protetta. Parco Adda Sud. Conoscere il Parco, 6, 206 pp.

Locasciulli O., NAPOlEONe I., PAlladino S., 1999 - Le aree italiane con provvedimento di tutela (al 31 dicembre 1998). CNR, Gruppo di studio sulle aree protette, Roma.

LoRENZETTI E., BATTISTI C., 2006 - Area as component of habitat fragmentation: corroborating its role in breeding bird communities and guilds of oak wood fragments in Central Italy. Revue d'Ecologie (Terre Vie), 61: 53-68.

LoREnZetTi E., BAtTisti C., 2007 - Nature reserve selection on forest fragments in a suburban landscape (Rome, Central Italy): indications from a set of avian species. Landscape Research, 32: 57-78.

MARI Z., 2005 - Il Parco naturale-archeologico dell'Inviolata: una realtà da costruire. In: CALAMITA, U. (a cura di), Atti del Convegno di Studi "Parco archeologico naturale dell'Inviolata di Guidonia, le ragioni di una tutela", Guidonia, 25 gennaio 2003, pp. 69-85. Associazione culturale onlus Amici dell'Inviolata Guidonia, Provincia di Roma.

Massa B., Fontana P., Buzzetti F. M., Kleukers R., Odé B., 2012 - Orthoptera. Fauna d’Italia. XLVIII. Calderini - Edizioni Calderini de Il Sole 24 ORE S.p.A., Bologna.

Mazzei P., Morel D., Panfili R., 2019 - Moths and Butterflies of Europe and North Africa, databese online, www.leps.it.

Montelucci G., 1941 - La vegetazione dei dintorni di Guidonia. In: Carella V., Note geologiche e storiche sul territorio cornicolano. Min. Aeron. Stabil. Fotomecc., Roma.

Pesarini C., Monzini V., 2011 - Insetti della Fauna Italiana. Coleotteri Carabidi II. Natura. Società Italiana di Scienze Naturali - Museo Civico di Storia Naturale di Milano - Civico Planetario "Ulrico Hoepli” - Acquario Civico di Milano, Milano, 101: 3-144.

Riservato E., Festi A., Fabbri R., Grieco C., Hardensen S., La Porta G., Landi F., Siesa M. E., Utzeri C., 2014 - Atlante delle libellule italiane, preliminare. Società Italiana per lo Studio e la Conservazione delle Libellule. Edizioni Belvedere, Latina, "Le Scienze" (17), 224 pp.

Sbrenna G., Micciareldi Sbrenna A., 2008 - Le Termiti italiane. Catalogo topografico e considerazioni zoogeografiche (Isoptera). Memorie della Società Entomologica Italiana, 87: 33-60.

Sтосн F., 2003 - Checklist of the species of the italian fauna. Online version 2.0.

Sтосн F., 2005 - Piccole acque (pp. 11-27). In: Stoch F. (ed.). Pozze, stagni e paludi. Le piccole acque, oasi di biodiversità. Quaderni Habitat, $\mathrm{n}^{\circ}$ 11, Ministero dell'Ambiente e della Tutela del Territorio, Museo Friulano di Storia Naturale, Comune di Udine, $158 \mathrm{pp}$.

Utzeri C., Dell'Anna L., 1997 - Odonata, pp. 75-78. In: M. Zapparoli (ed.), Gli Insetti di Roma. Comune di Roma, Dip. X Area Risorsa Suolo e Tutela Ambiente, Quaderni dell'Ambiente, 6.

Wizen G., Gasith A., 2011 - An Unprecedented Role Reversal: Ground Beetle Larvae (Coleoptera: Carabidae) Lure Amphibians and Prey upon Them. PLoS ONE 9: e25161.

Zanetti A., Tagliapietra A., 2004 - Studi sulle taxocenosi a Staphylininae in boschi di latifoglie italiani (Coleoptera, Staphylinidae). Studi Trentini Scienze Naturali, Acta Biologica, 81: 207-231.

ZANGHERI P., 2001 - Il naturalista esploratore raccoglitore preparatore imbalsamatore. Sesta edizione riveduta (con un capitolo sull'allestimento dei piccoli musei scolastici di storia naturale). Editore Ulrico Hoepli, Milano, 503 pp.

Zapparoli M. (ed.), 1997 - Gli Insetti di Roma. Comune di Roma, Dip. X Area Risorsa Suolo e Tutela Ambiente, Quaderni dell'Ambiente, 6 . 
Checklist di alcuni gruppi selezionati dell'entomofauna del Parco Naturale Archeologico dell'Inviolata

Appendice: Checklist di Insecta del Parco Naturale Archeologico dell'Inviolata.

Tab. A1. Lista di Odonata del PNA Inviolata.

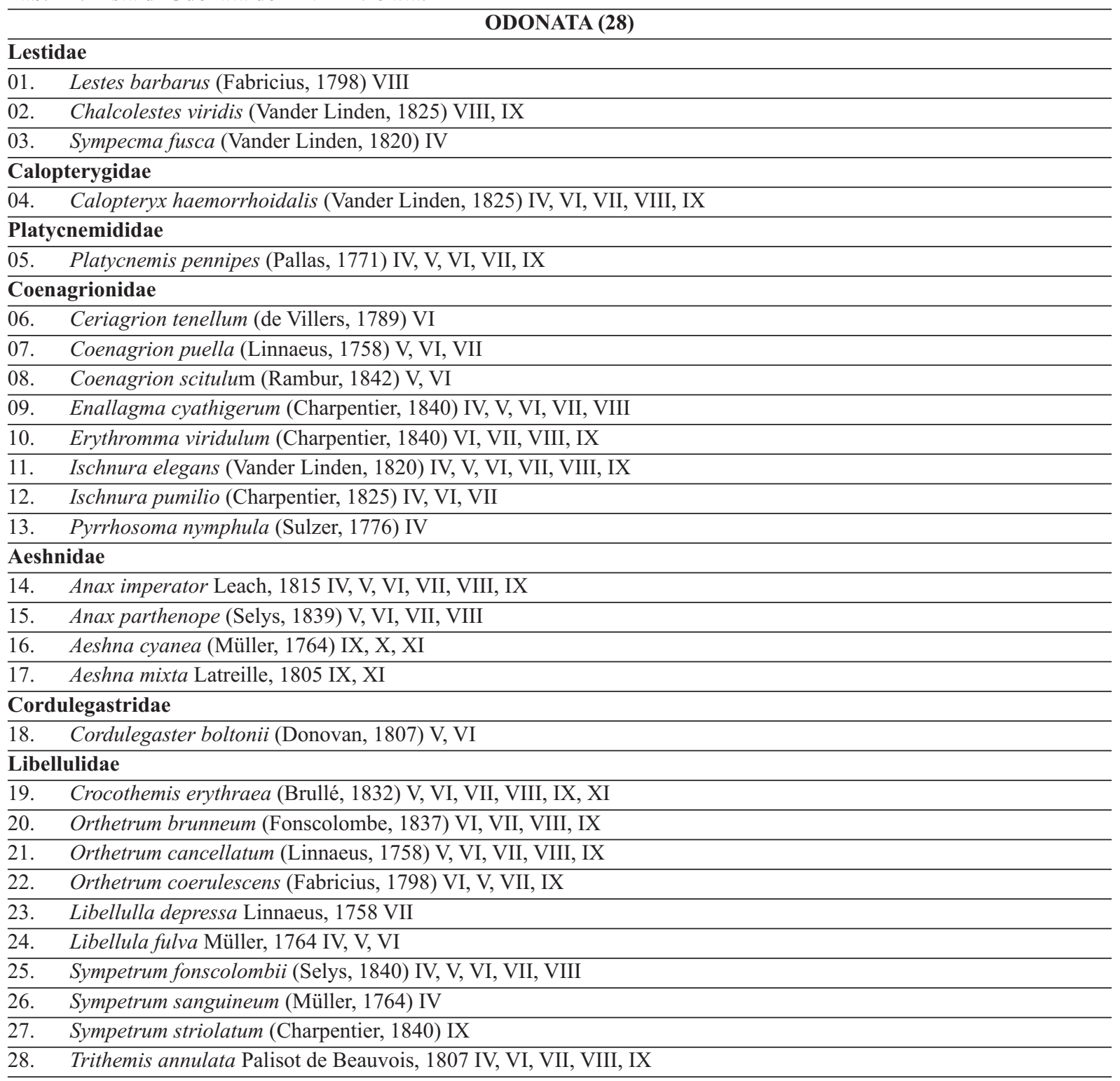

Tab. A2. Lista di Orthopteroidaea e Dermaptera del PNA Inviolata.

\section{MANTODEA (5)}

\begin{tabular}{ll}
\hline \multicolumn{2}{l}{ Mantidae } \\
\hline 01. & Ameles decolor (Charpentier, 1825) VII-VIII \\
\hline 02. & Ameles spallanziana (Rossi, 1792) VIII, IX, X \\
\hline 03. & Iris oratoria (Linnaeus, 1758) IX, X \\
\hline 04. & Mantis religiosa (Linnaeus, 1758) VII-X \\
\hline
\end{tabular}

Continua nella pagina seguente 
Tab. A2. Segue dalla pagina precedente

Empusidae

05. $\quad$ Empusa pennata (Thunberg, 1815) VIII, X

\section{ISOPTERA (2)}

\section{Kalotermitidae}

01. Kalotermes flavicollis (Fabricius, 1793) III, XI

Rhinotermitidae

02. Reticulitermes lucifugus (Rossi, 1792) II

\section{ORTHOPTERA (25)}

Tettigoniidae

01. Eupholidoptera chabrieri chabrieri Charpentier, $1825 \mathrm{VI}$

02. Phaneroptera nana Fieber, 1853 IX, X

03. Platycleis affinis affinis Fieber, $1853 \mathrm{VI}, \mathrm{VIII}$

04. Ruspolia nitidula (Scopoli, 1786) IX

05. Tettigonia viridissima Linnaeus, 1758 IV, V, VI

\section{Rhaphidophoridae}

06. Dolichopoda geniculata geniculata (O. G. Costa, 1836) III, IV, VI, VII, VIII, IX, XI E

\section{Gryllidae}

07. Eumodicogryllus bordigalensis bordigalensis (Latreille, 1804) VII

08. Gryllomorpha (Gryllomorpha) dalmatina dalmatina Ocskay, 1832 VIII

09. Melanogryllus desertus desertus (Pallas, 1771) III, V

\section{Mogoplistidae}

10. Mogoplistes brunneus Serville, 1839 VII

\section{Gryllotalpidae}

11. Gryllotalpa gryllotalpa (Linnaeus, 1758) IV, V, VII

\section{Tetrigidae}

12. Depressotetrix depressa (Brisout de Barneville, 1848) III

13. Tetrix subulata (Linnaeus, 1758) III

\section{Acrididae}

14. Acrida ungarica mediterranea Dirsh, 1949 VIII, IX

15. Acrotylus patruelis (Herrich - Schaeffer, 1838) IX

16. Aiolopus strepens strepens (Latreille, 1804) II, III, VIII

17. Aiolopus thalassinus thalassinus (Fabricius, 1781) IX

18. Anacridium aegyptium (Linnaeus, 1764) IV, V, VI, VII, IX, X

19. Calliptamus italicus italicus (Linnaeus, 1758) VIII

20. Chorthippus (Glyptobothrus) brunneus brunneus (Thunberg, 1815) VII

21. Locusta migratoria cinerascens Fabricius, 1781 VII, VIII, IX

22. Oedipoda caerulescens caerulescens (Linnaeus, 1758) VII, VIII, IX

23. Oedipoda germanica germanica (Latreille, 1804) VII

24. Omocestus (Omocestus) rufipes (Zetterstedt, 1821) IX

25. Pezotettix giornae (Rossi, 1794) IX

\section{PHASMATODEA (1)}

\section{Bacillidae}

01. Bacillus rossius (Rossi, 1788) IX, X

\section{DERMAPTERA (5)}

\section{Anisolabididae}

01. Euborellia moesta (Gené, 1837) II, III, IV, V, VI, IX, XII 
Checklist di alcuni gruppi selezionati dell'entomofauna del Parco Naturale Archeologico dell'Inviolata

Tab. A2. Segue dalla pagina precedente

Forficulidae

02. Forficula auricularia Linnaeus, 1758 I, II, III, V, VI, VIII, IX

03. Forficula decipiens Gené, 1832 III

Labiduridae

04. $\quad$ Nala lividipes (Dufour, 1828) IX

Spongiphoridae

05. Labia minor (Linnaeus, 1758) IX

Tab. A3. Lista di Coleoptera Carabidae del PNA Inviolata.

COLEOPTERA Carabidae (77)

01. Acinopus (Acinopus) picipes (Olivier, 1795) VII

02. Acupalpus (Acupalpus) maculatus (Schaum, 1860) III

03. Agonum (Agonum) marginatum (Linnaeus, 1758) II, III, VI, VIII, X

04. Agonum (Melanagonum) lugens (Duftschmid, 1812) I, II

05. Agonum (Punctagonum) viridicupreum (Goeze, 1777) I, III, VI

06. Agonum (Agonum) nigrum Dejean $1828 \mathrm{X}$

07. Agonum (Agonum) sordidum Dejean 1828 IX

08. Agonum (Olisares) permoestum Puel, 1938 cf. III

09. Amara (Amara) aenea (De Geer, 1774) VII, IX

10. Anchomenus (Anchomenus) dorsalis (Pontoppidan, 1763) II, III, IV

11. Anisodactylus (Anisodactylus) binotatus (Fabricius, 1787) III, VI

12. Asaphidion stierlini (Heyden, 1880) VI

13. Bembidion quadripustulatum quadripustulatum Audinet-Serville 1821 VIII

14. Brachinus (Brachinoaptinus) italicus (Dejean, 1831) I, II, III, IX, XI, XII E

15. Brachinus (Brachinus) crepitans (Linnaeus, 1758) I, IX, X, XII,

16. Brachinus (Brachinus) plagiatus Reiche, 1868 IV, V, VI, VII, IX

17. Brachinus (Brachynidius) sclopeta (Fabricius, 1792) II, X, XI

18. Bradycellus (Bradycellus) verbasci (Duftschmid, 1812) I, II, VI

19. Calathus (Neocalathus) cinctus Motschulsky, 1850 III

20. Calathus (Calathus) fuscipes graecus Dejean, 1831 III, IV, V, VI, VIII, IX, X

21. Calosoma (Calosoma) sycophanta (Linnaeus, 1758)

22. Calosoma (Campalita) maderae (Fabricius, 1775) VIII, IX

23. Carabus (Archicarabus) rossii Dejean, 1826 I, XI E

24. Carabus (Megodontus) violaceus picenus A. \& G. B. Villa, 1838 IV, V, IX, X

25. Carabus (Procrustes) coriaceus coriaceus Linnaeus, 1758 VIII

26. Carterus (Sabienus) tricuspidatus (Fabricius, 1792) VII, VIII

27. Chlaeniellus nigricornis (Fabricius 1787) III, V

28. Chlaeniellus olivieri (Crotch, 1871) II, III, VI, IX

29. Chlaeniellus vestitus (Paykull, 1790) I, II, III, V, VI, VIII, IX, X

30. Chlaenius (Chlaenites) spoliatus (Rossi, 1792) III, IV, VI, VII, VIII, IX, X

31. Chlaenius (Chlaenius) festivus (Panzer, 1796) III, VII, VIII, IX

32. Cicindela (Cicindela) campestris campestris Linnaeus, 1758 III, IV

33. Demetrias (Demetrias) atricapillus (Linnaeus, 1758) VI

34. Dinodes (Dinodes) decipiens (L. Dufour, 1820) III

35. Ditomus clypeatus (Rossi, 1790) VI

36. Dromius (Dromius) meridionalis Dejean, 1825 XII 
Tab. A3. Segue dalla pagina precedente

37. $\quad$ Drypta (Drypta) dentata (Rossi, 1790) I, II

38. Dyschiriodes sp.

39. $\quad$ Epomis circumscriptus (Duftschmid, 1812) IV, V, VIII

40. Harpalus (Harpalus) attenuatus Stephens, 1828 III

41. Harpalus (Harpalus) atratus Latreille, $1804 \mathrm{X}$

42. Harpalus (Harpalus) cupreus cupreus Dejean, $1829 \mathrm{VIII}$

43. Harpalus (Harpalus) dimidiatus (Rossi, 1790) IV, V, IX

44. Harpalus (Harpalus) distinguendus (Duftschmid, 1812) II, IV

45. Harpalus (Harpalus) serripes (Quensel in Schonherr, 1806) III

46. Harpalus (Harpalus) sulphuripes Germar, 1824 VII

47. Laemostenus (Actenipus) latialis Leoni, 1907 III, IV E

48. Lamprias fulvicollis (Fabricius, 1792) II

49. Lebia scapularis (Fourcroy, 1785) III, V, VIII, XI

50. $\quad$ Leistus (Leistus) fulvibarbis Dejean, 1826 III

51. Licinus (Licinus) silphoides (Rossi, 1790) X

52. Nebria (Nebria) brevicollis (Fabricius, 1792) II, III, IV, V, X

53. Notiophilus geminatus Dejean \& Boisduval, $1830 \mathrm{~V}, \mathrm{XI}$

54. $\quad$ Notiophilus rufipes Curtis, 1829 I, VIII, IX

55. Notiophilus substriatus G. R. Waterhouse, 1833 I

56. Ocydromus (Nepha) genei illigeri (Netolitzky 1914) V

57. Ocys harpaloides (Audinet-Serville, 1821) IV

58. Ophonus (Metophonus) puncticeps Stephens, $1828 \mathrm{cf}$. IX

59. Paradromius (Manodromius) linearis (Olivier, 1795) VI

60. $\quad$ Paranchus albipes (Fabricius, 1796) II, III, IX, X

61. Paratachys fulvicollis (Dejean, 1831) III

62. Platyderus (Platyderus) neapolitanus (Reiche, 1855) III, VIII, IX E

63. Poecilus (Poecilus) cupreus (Linnaeus, 1758) V, XI

64. Poecilus (Poecilus) cursorius (Dejean, 1828) VIII

65. Pseudoophonus (Pseudoophonus) rufipes (De Geer, 1774) III, V, VI, VII, VIII, IX, X, XII

66. Pterostichus (Adelosia) macer (Marsham, 1802) XII

67. Pterostichus (Argutor) cursor (Dejean, 1828) I, II

68. Pterostichus (Feronidius) melas italicus (Dejean, 1828) III, IV, IX, X, XI, XII

69. Pterostichus (Pseudomaseus) nigrita (Paykull, 1790) I, II, III, VIII, IX, X, XI

70. Pterostichus (Pterostichus) micans Heer, 1841 IV, IX

71. Scybalicus oblongiusculus (Dejean, 1829) VI, IX, XII

72. Siagona europaea Dejean, $1826 \mathrm{X}$

73. Stenolophus (Stenolophus) teutonus (Schrank, 1781) III, V

74. Tachyura (Tachyura) diabrachys (Kolenati, 1845) cf. VIII

75. $\quad$ Trechus (Trechus) quadristriatus (Schrank, 1781) VI, IX

76. Trepanes (Trepanes) articulatus (Panzer, 1796) VIII, IX, X

77. Zabrus (Zabrus) tenebrioides tenebrioides (Goeze, 1777) VIII, IX, X, XI

Tab. A4. Lista di Coleoptera (Carabidae esclusi) del PNA Inviolata.

COLEOPTERA (Carabidae esclusi) (282)

\section{Haliplidae}

01. Haliplus (Haliplus) lineaticollis (Marsham, 1802) IV

02. Peltodytes rotundatus (Aubé, 1836) IV 
Checklist di alcuni gruppi selezionati dell'entomofauna del Parco Naturale Archeologico dell'Inviolata

Tab. A4. Segue dalla pagina precedente

Gyrinidae

03. Gyrinus (Gyrinus) marinus Gyllenhal, $1808 \mathrm{cf}$. IV

04. Gyrinus (Gyrinus) urinator Illiger, 1807 I, III, IV, IX, X

\section{Dytiscidae}

05. Agabus (Gaurodytes) bipustulatus (Linnaeus 1767) III, IV

06. Agabus (Gaurodytes) didymus (Olivier, 1795) VI

07. $\quad$ Agabus (Gaurodytes) nebulosus (Forster, 1771) III, IV, V

08. Colymbetes fuscus (Linnaeus, 1758) II, XII

09. Hydroglyphus geminus (Fabricius, 1792) cf. VI, VII

10. Hygrotus (Coelambus) confluens (Fabricius, 1787) VI

11. Hyphydrus aubei Ganglbaueri, 1892 VIII

12. Laccophilus hyalinus (De Geer, 1774) III, IV

13. Rhantus (Rhantus) suturalis (MacLeay, 1825) III, VI

\section{Hydrophilidae}

14. Cercyon sp. III

15. Coelostoma (Coelostoma) hispanicum (Kuster 1848) I, II, X

16. Helochares sp. II, X

17. Hydrochara caraboides (Linnaeus, 1758) II, III

18. Laccobius sp. II

19. Sphaeridium sp. III

\section{Histeridae}

20. $\quad$ Hister quadrimaculatus Linnaeus, 1758 cf. III, IV, VIII, IX

\section{Silphidae}

21. Ablattaria laevigata (Fabricius, 1775) IV, VII, IX

22. Nicrophorus interruptus Stephens, $1830 \mathrm{~V}$

23. Nicrophorus vestigator Herschel, $1807 \mathrm{VI}$

24. Phosphuga atrata atrata (Linnaeus, 1758) IV, X

25. Silpha obscura Linnaeus, 1758 II, III, IV

26. Silpha olivieri Bedel, 1887 III

27. Silpha tristis Illiger, $1798 \mathrm{~V}, \mathrm{VII}$

28. Thanatophilus sinuatus (Fabricius, 1775) III, V

\section{Scydmaenidae}

29. Cephennium sp. Müller \& Kunze, 1822 XI

30. Leptomastax hypogea Pirazzoli, 1855 IX

\section{Staphylinidae}

31. Creophilus maxillosus (Linnaeus, 1758) III, V, IX

32. Ocypus (Matidus) italicus italicus (Aragona, 1830) IV, VIII, IX, XII E

33. Ocypus (Matidus) nitens (Schrank, 1781) I, II, III

34. Ocypus (Ocypus) olens (O. F. Müller, 1764) IX, X, XI

35. Ocypus (Ocypus) ophthalmicus (Scopoli, 1763) VIII

36. Ocypus (Pseudocypus) sericeicollis (Ménétriés, 1832) cf. XI

37. Ontholestes murinus (Linnaeus, 1758) IV

38. Othius punctulatus (Goeze, 1777) XII

39. $\quad$ Paederus littoralis (Gravenhorst, 1802) cf. I, II, III, X

40. $\quad$ Platydracus (Platydracus) stercorarius (Olivier, 1795) X

41. Quedius (Quedius) molochinus (Gravenhorst, 1806) cf. X

42. $\quad$ Staphylinus dimidiaticornis Gemminger, $1851 \mathrm{IV}, \mathrm{V}, \mathrm{X}$ 
Tab. A4. Segue dalla pagina precedente

43. Tasgius (Rayacheila) falcifer (Nordmann, 1837) II

44. Tasgius (Rayacheila) winkleri (Bernhauer, 1906) VIII, X, XI

45. Tasgius (Tasgius) pedator (Gravenhorst, 1802) IV, VIII, IX

\section{Ptiliidae}

46. $\quad$ Nossidium sp. XII

Lucanidae

47. $\quad$ Dorcus parallelipipedus parapellelipipedus (Linnaeus, 1785) IV, VIII

48. Lucanus (Lucanus) tetraodon Thunberg 1806 VIII, IX, X

\section{Trogidae}

49. Trox litoralis Pittino, 1991 I, II

50. Trox niger Rossi, 1792 IV, V, IX

\section{Geotrupidae}

51. Jekelius (Jekelius) intermedius (Costa, 1827) III, IV, V, IX, X

\section{Aphodiidae}

52. Acrossus luridus (Fabricius, 1775) III, IV, V

53. Agrilinus convexus (Erichson, 1848) I, III

54. Aphodius foetidus (Herbst, 1783) IV, V

55. Biralus mahunkaorum Ádám, 1983 IV

56. Chilothorax lineolatus (Illiger, 1803) I, IV, IX

57. Colobopterus erraticus (Linnaeus, 1758) IV

58. Loraphodius suarius (Faldermann, 1835) IX

59. Melinopterus consputus (Creutzer, 1799) II

60. Melinopterus prodromus (Brahm, 1790) I, III

61. Nialus varians (Duftschmid 1805) IV

62. Phalacronothus biguttatus (Germar 1824) V

63. Phalacronothus quadrimaculatus quadrimaculatus (Linnaeus, 1761) V

64. Pleurophorus caesus (Creutzer in Panzer, 1796) VI

\section{Scarabaeidae}

65. Bubas bison (Linnaeus 1767) III, IV

66. Caccobius schreberi (Linnaeus 1758) IV

67. Copris hispanus (Linnaeus, 1764) III

68. Euoniticellus fulvus (Goeze 1777) IV, V

69. Onthophagus (Onthophagus) taurus (Schreber, 1759) IV, IX

70. Onthophagus (Palaeonthophagus) coenobita (Herbst, 1783) IV, XI

71. Onthophagus (Palaeonthophagus) opacicollis Reitter, 1892 III, IV

72. Onthophagus (Palaeonthophagus) ruficapillus Brullè, 1832 III, IV, V, VI

73. Onthophagus (Palaeonthophagus) vacca (Linnaeus, 1767) IV

\section{Glaphyridae}

74. Amphicoma carceli (Laporte, 1832) V E

\section{Melolonthidae}

75. Melolontha pectoralis Megerle, 1812 IV

76. Aplidia etrusca Kraatz, 1882 VI E

\section{Dynastidae}

77. Oryctes nasicornis (Linnaeus, 1758) VI

78. Pentodon bidens punctatus (Villers, 1789) III, V, VII, VIII, IX, X, XII

\section{Cetoniidae}

79. Cetonia aurata pisana (Linnaeus, 1761) IV, V, VI 
Checklist di alcuni gruppi selezionati dell'entomofauna del Parco Naturale Archeologico dell'Inviolata

Tab. A4. Segue dalla pagina precedente

80. $\quad$ Oxythyrea funesta (Poda, 1761) IV, V, VI, XII

81. Protaetia (Netocia) morio morio (Fabricius, 1781) V, VI

82. Protaetia (Netocia) cuprea (Fabricius, 1775) IV, V, VI

83. Trichius gallicus gallicus Dejean, $1821 \mathrm{~V}$

84. Tropinota (Epicometis) hirta (Poda, 1761) IV

85. Valgus hemipterus (Linnaeus, 1758) IV, V, VI

\section{Heteroceridae}

86. Heterocerus sp. X

Dryopidae

87. $\quad$ Dryops sp. I, II

88. Pomatinus substriatus (Müller, 1806) IV

\section{Elateridae}

89. Agriotes (Agriotes) lineatus (Linnaeus, 1767) IV

90. Agriotes (Agriotes) litigiosus (Rossi, 1792) V, VI, VIII

91. Agriotes (Agriotes) sordidus (Illiger, 1807) I, IV, V, VI

92. Ampedus (Ampedus) cardinalis (Schiodte, 1865) I

93. Ampedus (Ampedus) praeustus (Fabricius, 1792) I, II

94. Athous (Athous) limoniiformis Candèze, 1865 VI

95. Athous (Athous) vittatus (Gmelin, 1790) IV, V

96. Cardiophorus vestigialis Erichson, $1840 \mathrm{cf}$. IV

97. Cardiophorus goezi Sanchez - Ruiz, 1996 XII

98. Cardiophorus italicus Platia \& Bartolozzi, 1988 I, V, XII

99. Drasterius bimaculatus (Rossi, 1790) V

100. Hemicrepidius hirtus (Herbst, 1784) V

101. Lacon punctatus (Herbst, 1779) I, II, VI, XII

102. Melanotus (Melanotus) dichrous (Erichson, 1841) VI

103. Melanotus (Melanotus) tenebrosus (Erichson, 1841) VI

104. Quasimus (Quasimus) minutissimus (Germar, 1822) VI, VII, XI

105. Synaptus filiformis (Fabricius, 1781) V, VI

\section{Cebrionidae}

106. Cebrio (Cebrio) dubius (Rossi, 1790) cf. IX E

\section{Buprestidae}

107. Agrilus graminis Gory \& Laporte, $1837 \mathrm{~V}$

108. Agrilus viridis Linnaeus $1758 \mathrm{~V}$

109. Agrilus lineola lineola Kiesenwetter, $1857 \mathrm{~V}$

110. Agrilus laticornis (Illiger, 1803) V

111. Agrilus obscuricollis Kiesenwetter, $1857 \mathrm{~V}$

112. Agrilus viridicaerulans rubi Schaefer, 1937 VI

113. Anthaxia (Anthaxia) lucens Kuster, $1852 \mathrm{~V}$

114. Anthaxia (Anthaxia) nitidula (Linnaeus, 1758) IV

115. Anthaxia (Anthaxia) thalassophila thalassophila Abeille de Perrin, 1900 VI, IX

116. Anthaxia (Cratomerus) hungarica hungarica (Scopoli, 1772) V

117. Anthaxia (Haplantaxia) millefolii polychloros Abeille de Perrin V, VI, VII

118. Anthaxia (Haplanthaxia) scutellaris scutellaris Gené, 1894 IV

119. Capnodis tenebricosa (Olivier, 1790) X

120. Capnodis tenebrionis (Linnaeus, 1758) VI

121. Chrysobothris (Chrysobothris) affinis affinis (Fabricius, 1794) VII 
Tab. A4. Segue dalla pagina precedente

122. Coraebus rubi (Linnaeus, 1767) VI, VII, VIII

123. Eurythyrea micans (Fabricius, 1794) VII

123. Lamprodila mirifica (Mulsant, 1855) VI, VII

125. Trachys minutus (Linnaeus, 1758) V

126. Trachys troglodytiformis Obenberger, $1918 \mathrm{~V}$

Drilidae

127. Drilus flavescens (Olivier, 1790) IV

Lampyridae

128. Lampyris noctiluca (Linnaeus, 1767) VI

129. Luciola (Luciola) lusitanica (Charpentier, 1825) VI

\section{Cantharidae}

130. Cantharis (Cantharis) fusca Linnaeus, 1758 IV

131. Rhagonycha (Rhagonycha) fulva (Scopoli, 1763) IV

\section{Dermestidae}

132. Dermestes (Dermestinus) frischii Kugelann, 1792 IV

133. Dermestes (Dermestinus) undulatus Brahm, $1790 \mathrm{IV}, \mathrm{V}$

\section{Trogossitidae}

134. Temnochila caerulea (Olivier, 1790) III, XI, XII

135. Tenebroides mauritanicus Linnaeus, 1758 III, XI

\section{Cleridae}

136. Denops albofasciatus (Charpentier, 1825) V

137. Necrobia rufipes (De Geer, 1775) IX

138. Thanasimus formicarius (Linnaeus, 1758) III

139. Trichodes alvearius (Fabricius, 1792) IV, V, VI

\section{Dasytidae}

140. Dolichosoma simile (Brullé, 1832) V

141. Enicopus sp. VI

142. Psilothrix viridicoerulea (Geoffroy, 1785) IV, V

\section{Malachiidae}

143. Clanoptilus (Clanoptilus) rufus (Olivier, 1790) V

144. Clanoptilus (Clanoptilus) spinipennis (Germar, 1824) V

145. Malachius australis Mulsant \& Rey, 1867 IV

\section{Nitidulidae}

146. Brassicogethes aeneus (Fabricius, 1775) III

\section{Silvanidae}

147. Oryzaephilus surinamensis (Linnaeus, 1758) III

148. Uleiota planata (Linnaeus, 1761) I, II, III, XI

\section{Cryptophagidae}

149. Atomaria (Atomaria) gravidula Erichson, 1846 XI, XII

150. Cryptophagus sp. XII

\section{Languriidae}

151. Cryptophilus integer (Heer, 1841) XI

\section{Erotylidae}

152. Triplax lacordairii Crotch, 1870 I

\section{Coccinellidae}

153. Adalia (Adalia) bipunctata bipunctata (Linnaeus, 1758) V

154. Coccinella (Coccinella) septempunctata Linnaeus, 1758 III, IV, V, VI, IX, X 
Checklist di alcuni gruppi selezionati dell'entomofauna del Parco Naturale Archeologico dell'Inviolata

Tab. A4. Segue dalla pagina precedente

155. Harmonia axyridis Pallas, $1773 \mathrm{~V}$ A

156. Oenopia conglobata (Linnaeus, 1758) III, X

157. Propylea quatuordecimpunctata (Linnaeus, 1758) IV

Laemophloeidae

158. Cryptolestes duplicatus (Walt1, 1834) XII

159. Cryptolestes ferrugineus Stephens, 1830 III, XII

\section{Latridiidae}

160. Corticaria elongata (Gyllenhal, 1827) VIII

161. Enicmus transversus (Olivier, 1790) XI, XII

162. Migneauxia crassiuscula (Aubé, 1850) III

\section{Mycetophagidae}

163. Litargus connexus (Geoffroy, 1785) XII

164. Typhaea stercorea (Linnaeus, 1758) III, VIII

\section{Ripiphoridae}

165. Macrosiagon ferrugineum (Fabricius, 1775) VIII

\section{Meloidae}

166. Lytta (Lytta) vesicatoria (Linnaeus, 1758$) \mathrm{V}$

167. Meloe (Meloe) proscarabaeus proscarabaeus Linnaeus, 1758 III

168. Meloe (Eurymeloe) mediterraneus G. Müller, 1925 IX, X, XI

169. Meloe (Treiodes) autumnalis autumnalis Olivier, $1792 \mathrm{X}, \mathrm{XI}$

\section{Oedemeridae}

170. Oedemera sp. V

\section{Pyrochroidae}

171. Pyrochroa serraticornis (Scopoli, 1763) IV

\section{Anthicidae}

172. $\quad$ Anthelephila pedestris (Rossi, 1790) I

173. Anthicus laeviceps Baudi di Selve, 1877 IV

174. Hirticollis hispidus (Rossi, 1792) VII

175. $\quad$ Stricticollis tobias (Marseul, 1879) cf. VIII

176. $\quad$ Notoxus trifasciatus Rossi, 1792 VI

\section{Tenebrionidae}

177. Accanthopus velikensis (Piller \& Mitterpacher, 1783) I, II, VI, VIII, XII

178. Alphitobius diaperinus (Panzer, 1797) I, II

179. $\quad$ Blaps gibba Laporte de Castelnau, 1840 I, IV, VII, VIII, IX, XII

180. Colpotus strigosus strigosus (A. Costa 1847) I, III, IX, X E

181. Corticeus (Corticeus) bicolor (A. G. Olivier, 1790) XII

182. Diaperis boleti (Linnaeus, 1758) I

183. Gonocephalum (Gonocephalum) granulatum nigrum (Küster, 1849) VII

184. Hypophloeus unicolor (Piller \& Mitterpacher, 1783) II

185. Lagria hirta (Poda, 1761) V, VI, IX

186. Omophlus sp. IV

187. Nalassus dryadophilus (Mulsant, 1854) VI, IX

188. Pedinus meridianus Mulsant \& Rey, 1853 III, IV, VII, VIII

189. Platydema violaceum (Fabricius, 1790) XII

190. Scaurus striatus Fabricius, 1792 I, IV, XII

191. Stenosis sp. III

192. Uloma culinaris (Linnaeus, 1758) I 
Tab. A4. Segue dalla pagina precedente

\section{Zopheridae}

193. Corticus celtis Germar, 1824 XII

Cerambycidae

194. Agapanthia (Agapanthia) cardui (Linnaeus, 1767) IV, V

195. Agapanthia (Epoptes) villosoviridescens (De Geer, 1775) IV

196. Aromia moschata moschata (Linnaeus, 1758) VII

197. Calamobius filum (Rossi, 1790) IV, V, VI

198. Cerambyx scopolii Fuessly, 1775 IV, V, VI

199. Clytus arietis (Linnaeus, 1758) V

200. Deilus fugax (Olivier, 1790) V

201. Herophila tristis (Linnaeus 1767) V, XI

202. Mesosa nebulosa (Fabricius 1781) I, V, VI, VII

203. Grammoptera ruficornis ruficornis (Fabricius, 1781) IV

204. Oberea linearis (Linnaeus, 1761) V

205. Opsilia coerulescens (Scopoli, 1763) IV

206. Paracorymbia fulva (De Geer, 1775) V, VI

207. Plagionotus scalaris (Brullé 1832) V, VI

208. Phymatodes testaceus (Linnaeus, 1758) V

209. Phytoecia virgula (Charpentier, 1825) V

210. Prionus coriarius (Linnaeus, 1758) VI

211. Pseudovadonia livida livida (Fabricius, 1776) V

212. Purpuricenus kaehleri kaehleri (Linnaeus, 1758) VII

213. Ropalopus clavipes (Fabricius, 1775) V

214. Rutpela maculata (Poda, 1761) V, VI

215. Saperda punctata (Linnaeus, 1767) V, VI, VII

216. Stenhomalus (Obriopsis) bicolor (Kraatz, 1862) IV

217. Stenopterus ater Linnaeus, 1767 VI, VII

218. Stenopterus rufus Linnaeus, $1767 \mathrm{~V}$

219. Stenurella melanura (Linnaeus, 1758) VI

220. Stictoleptura cordigera (Fuessly, 1775) VI

221. Vesperus luridus (Rossi, 1794) VIII, IX

222. Xylotrechus arvicola (Olivier, 1795) VI

223. Xylotrechus stebbingi Gahan, 1906 VI A

\section{Chrysomelidae}

224. Altica quercetorum Foudras, $1860 \mathrm{XI}$

225. Cassida (Cassida) rubiginosa O. F. Müller, $1776 \mathrm{~V}$

226. Cassida (Cassida) vibex Linnaeus, 1767 IV

227. Cheilotoma (Cheilotoma) erythrostoma italica Leoni, $1906 \mathrm{~V}$

228. Chrysomela (Chrysomela) populi Linnaeus, 1758 IX

229. Chrysolina (Chrysolina) bankii (Fabricius, 1775) III, IV, VI, IX, XII

230. Chrysolina (Colaphodes) haemoptera (Linnaeus, 1758) IX, X

231. Chrysolina (Fastuolina) fastuosa fastuosa (Scopoli, 1763) VI

232. Chrysolina (Melasomoptera) grossa (Fabricius, 1792) IV, IX, X

233. Chrysolina (Stichoptera) rossia (Illiger, 1802) III, VIII, X

234. Chrysolina (Synerga) herbacea (Duftschmid, 1825) IV, IX

235. Chrysolina (Taeniochrysea) americana Linnaeus, $1758 \mathrm{~V}$

236. Cryptocephalus (Cryptocephalus) marginatus Fabricius, $1781 \mathrm{~V}$ 
Checklist di alcuni gruppi selezionati dell'entomofauna del Parco Naturale Archeologico dell'Inviolata

Tab. A4. Segue dalla pagina precedente

237. Cryptocephalus sp. IV

238. Exosoma lusitanicum (Linnaeus, 1767) V

239. Galeruca (Galeruca) interrupta Illiger, $1802 \mathrm{VI}$

240. Galeruca (Emarhopa) rufa Germar, 1824 IV

241. Gonioctena (Spartomena) fornicata (Brüggemann, 1873) V A

242. Hispa atra Linnaeus, $1767 \mathrm{VI}$

243. Hypocassida subferruginea (Schrank, 1776) cf. V

244. Labidostomis (Labidostomis) taxicornis (Fabricius, 1792) V

245. Lachnaia (Lachnaia) italica italica (Weise, 1882) IV, V

246. Leptispa filiformis (Germar, 1842) $\mathrm{V}$

247. Timarcha (Timarcha) nicaeensis Villa, 1835 III, V, VI, VIII, XII

248. Oulema sp. III, IV

249. Xanthogaleruca luteola (O. F. Müller, 1766) I, II, V, VI, VIII

\section{Apionidae}

250. Ischnopterapion (Chlorapion) virens (Herbst 1797) IV

251. Protapion laevicolle (W. Kirby, 1811) XII

\section{Curculionidae}

252. Acallocrates minutesquamosus (Reiche, 1860) III

253. Aulacobaris cuprirostris (Fabricius, 1787) IV

254. Brachypera zoilus (Scopoli, 1763) IV

255. Cionus hortulanus (Fourcroy, 1785) cf. VII

256. Coelositona limosus (Rossi, 1792) IV

257. Curculio (Curculio) glandium Marsham, 1802 V, VI

258. Curculio (Curculio) villosus Fabricius, $1781 \mathrm{~V}$

259. Hypera (Hypera) postica (Gyllenhal, 1813) IV

260. Larinus (Larinomesius) obtusus Gyllenhal, 1835 VII

261. Larinus (Larinomesius) ochreatus (Olivier, 1807) VII

262. Larinus (Larinomesius) scolymi Olivier, $1807 \mathrm{VII}$

263. Lixomorphus algirus (Linnaeus, 1758) V

264. Lixus (Compsolixus) anguinus (Linnaeus, 1767) IV, V

265. Lixus (Dilixellus) pulverulentus (Scopoli, 1763) IV, V, VI, IX

266. Malvaevora timida (Rossi 1792) IV, V

267. Mogulones beckeri (Schultze, 1900) cf. IV

268. Otiorhynchus (Arammichnus) cribricollis Gyllenhal, 1834 I, III

269. Otiorhynchus (Nehrodistus) armatus Boheman, 1843 V E

270. Otiorhynchus (Zustalestus) rugosostriatus (Goeze, 1777) X

271. Phyllobius (Nemoicus) oblongus (Linnaeus 1758) V

272. Phyllobius (Phyllobius) etruscus Desbrochers, 1873 IV E

273. Polydrusus sp. IV

274. Rhinocyllus conicus (Frölich, 1792) I, II, III, IV, V, X, XI

275. Rhinusa tetra (Fabricius, 1792) VI

276. Rhytideres (Rhytideres) plicatus (Olivier, 1790) IX

277. Sitona (Sitona) ophthalmicus Desbrochers, 1869 III

278. Tychius (Tychius) quinquepunctatus (Linnaeus, 1758) III

279. Aclees sp. III, IV, VII, IX A

\section{Anthribidae}

280. Pseudeuparius centromaculatus (Gyllenhal, 1883) III 
Tab. A4. Segue dalla pagina precedente

Rhynchitidae

281. Tatianaerhynchites aequatus (Linnaeus, 1767) IV

Dryophthoridae

281. Rhyncophorus ferrugineus (Olivier, 1790) IV A

Tab. A5. Lista di Lepidoptera e Mecoptera del PNA Inviolata.

\section{LEPIDOPTERA (107)}

\section{Elachistidae}

01. Ethmia bipunctella (Fabricius, 1775) VI

\section{Oecophoridae}

02. Pleurota sp. VI

\section{Cossidae}

03. Cossus cossus (Linnaeus, 1758) XI

\section{Sesiidae}

04. Pyropteron chrisidiformis (Esper, 1782) VI

Zygaenidae

05. Zygaena (Zygaena) transalpina (Esper, 1780) VI

Tortricidae

06. Clepsis consimilana (Hübner, 1817) cf. VI

07. Cnephasia sp. VI

\section{Pyralidae}

08. Ematheudes punctella (Treitschke, 1833) VI

Crambidae

09. Nomophila noctuella (Denis \& Schiffermüller, 1775) V, VI

Thyrididae

10. Thyris fenestrella (Scopoli, 1763) V

\section{Lasiocampidae}

11. Eriogaster (Eriogaster) catax (Linnaeus, 1758) IX

12. Eriogaster (Eriogaster) lanestris (Linnaeus, 1758) IV

13. Lasiocampa (Lasiocampa) quercus (Linnaeus, 1758) VI

14. Lasiocampa (Pachygastria) trifolii (Denis \& Schiffermüller, 1775) IV, IX

\section{Sphingidae}

15. Agrius convolvuli (Linnaeus, 1758) IX

16. Deilephila porcellus (Linnaeus, 1758) VI

17. Laothoe populi (Linnaeus, 1758) VI

18. Macroglossum stellatarum (Linnaeus, 1758) III, IV, VI, IX, X

19. Mimas tiliae (Linnaeus, 1758) V

\section{Saturniidae}

20. Saturnia pavoniella (Scopoli, 1763) III, VI

\section{Hesperiidae}

21. Carcharodus alceae (Esper, 1780) III, VI

22. Ochlodes sylvanus (Esper, 1777) V

23. Thymelicus acteon (Rottemburg, 1775) VI

\section{Papilionidae}

24. Iphiclides podalirius (Linnaeus, 1758) IV, VII, VIII, IX

25. Papilio machaon Linnaeus, 1758 III, IV, V, VI, VII, VIII, IX 
Checklist di alcuni gruppi selezionati dell'entomofauna del Parco Naturale Archeologico dell'Inviolata

\section{Pieridae}

26. Anthocharis cardamines (Linnaeus, 1758) III, IV

27. Aporia crataegi (Linnaeus, 1758) V

28. Colias croceus (Fourcroy, 1785) IV, VI, VIII, IX

29. Euchloe ausonia (Hübner, 1805) III, IV, V, VI

30. Gonepteryx rhamni (Linnaeus, 1758) III, IV

31. Pieris brassicae (Linnaeus, 1758) IV, V

32. Pieris napi (Linnaeus, 1758) X

33. Pieris rapae (Linnaeus, 1758) III, V, VI, IX

\section{Lycaenidae}

34. Glaucopsyche (Glaucopsyche) alexis (Poda, 1761) IV

35. Lampides boeticus (Linnaeus, 1767) VIII, IX

36. Lycaena phlaeas (Linnaeus, 1761) IV, V

37. Lycaena thersamon (Esper, 1784) V, VI, IX

38. Lycaena tityrus (Poda, 1761) IV

39. Polyommatus (Polyommatus) icarus (Rottemburg, 1775) IV, V

40. Satyrium w-album (Knoch, 1782) VI

\section{Nymphalidae}

41. Aglais io (Linnaeus, 1758) V, VII

42. Argynnis (Argynnis) paphia (Linnaeus, 1758) IX

43. Charaxes jasius (Linnaeus, 1767) IX

44. Coenonympha pamphilus (Linnaeus, 1758) IV, VIII

45. Issoria (Issoria) lathonia (Linnaeus, 1758) V, VII

46. Lasiommata megera (Linnaeus, 1767) III, IV, V, VI, VII

47. Libythea celtis (Laicharting, 1782) V

48. Limenitis reducta Staudinger, 1901 IV, VI, VIII

49. Maniola jurtina (Linnaeus, 1758) IV, V, VI, IX

50. Melitaea athalia (Rottemburg, 1775) IV

51. Melitaea didyma (Esper, 1778) VI, VIII

52. Melitaea phoebe (Denis \& Shiffermuller, 1775) VII

53. Pararge aegeria (Linnaeus, 1758) VI, VII

54. Polygonia c-album (Linnaeus, 1758) V, VI, VIII

55. Vanessa atalanta (Linnaeus, 1758) I, III, IV, V, VI, VIII

56. Vanessa cardui (Linnaeus, 1758) III, IV, V, VII

\section{Geometridae}

57. Aspitates ochrearia (Rossi, 1794) IV, V

58. Ematurga atomaria (Linnaeus, 1758) III, IV

59. Hemithea aestivaria (Hübner, 1789) VI

60. Ligdia adustata (Denis \& Schiffermüller, 1775) VI

61. Lycia florentina (Stefanelli, 1882) V

62. Lycia hirtaria (Clerck, 1759) III

63. Menophra abruptaria (Thünberg, 1792) VI

64. Peribatodes rhomboidaria (Denis \& Shiffermuller, 1775) VI

65. Petrophora chlorosata (Scopoli, 1763) IV

66. Timandra comae Schmidt, $1931 \mathrm{~V}, \mathrm{VI}$

\section{Notodontidae}

67. Cerura (Cerura) vinula (Linnaeus, 1758) IV

68. Thaumetopoea pityocampa (Denis \& Schiffermüller, 1775) IV, VIII 
Tab. A5. Segue dalla pagina precedente

Erebidae

69. Amata phegea (Linnaeus, 1758) IV, V, VI

70. Arctia villica (Linnaeus, 1758) IV, V

71. Catocala nupta (Linnaeus, 1767) VI, VII, VIII

72. Coscinia striata (Linnaeus, 1758) V

73. Diacrisia sannio (Linnaeus, 1758) IX

74. Dysauxes famula (Freyer, 1836) cf. V, VI

75. Dysgonia algira (Linnaeus, 1767) cf. VI

76. Euplagia quadripunctaria (Poda, 1761) VI, VII, VIII

77. Hypena obsitalis (Hübner, 1813) III

78. Hypena palpalis (Hübner, 1796) VIII

79. Hypena rostralis (Linnaeus, 1758) VI

80. Lithosia quadra (Linnaeus, 1758) VI

81. Lymantria dispar (Linnaeus, 1758) VI, X

82. Orgyia (Orgyia) antiqua (Linnaeus, 1758) V

83. Phragmatobia fuliginosa (Linnaeus, 1758) IV

84. Spilosoma lubricipeda (Linnaeus, 1758) IV

85. Spilosoma lutea (Hufnagel, 1766) V

\section{Noctuidae}

86. $\quad$ Abrostola sp. VI

87. Acontia (Acontia) lucida (Hufnagel, 1766) VI

88. Acronicta (Triaena) psi (Linnaeus, 1758) VI

89. Acronicta (Viminia) rumicis (Linnaeus, 1758) IX

90. Agrotis bigramma (Esper, 1790) IX

91. Agrotis ipsilon (Hufnagel, 1766) VI

92. Agrotis puta (Hübner, 1803) IV

93. Anorthoa munda (Denis \& Schiffermüller, 1775) III

94. Autographa gamma (Linnaeus, 1758) III, IV, V, VIII

95. Conistra sp. II

96. Craniophora ligustri (Denis \& Schiffermüller, 1775) VI

97. Eremobia ochroleuca (Denis \& Schiffermüller, 1775) VI

98. Mamestra brassicae (Linnaeus, 1758) cf. IX

99. Mesapamea sp. IV

100. Mormo maura (Linnaeus, 1758) IX

101. Mythimna (Hyphilare) albipuncta (Denis \& Schiffermüller, 1775) IV

102. Mythimna (Mythimna) vitellina (Hübner, 1808) VIII

103. Noctua comes Hübner, $1813 \mathrm{VI}$

104. Noctua janthina (Denis \& Schiffermüller, 1775) VIII

105. Noctua pronuba (Linnaeus, 1758) VI

106. Polyphaenis sericata (Esper, 1787) VI

107. Tyta luctuosa (Denis \& Schiffermüller, 1775) IV, V, VI

MECOPTERA (1)

\section{Panorpidae}

01. Panorpa sp. IV, VI, IX 